\title{
A New Methodology for the Development of Numerical Methods for the Numerical Solution of the Schrödinger Equation
}

\author{
Z.A. Anastassi*, D.S. Vlachos ${ }^{\dagger}$ and T.E. Simos ${ }^{\ddagger}$ \\ Laboratory of Computational Sciences, Department of Computer Science and \\ Technology, Faculty of Sciences and Technology, University of Peloponnese, \\ GR-221 00 Tripolis, Greece
}

\begin{abstract}
In the present paper we introduce a new methodology for the construction of numerical methods for the approximate solution of the one-dimensional Schrödinger equation. The new methodology is based on the requirement of vanishing the phase-lag and its derivatives. The efficiency of the new methodology is proved via error analysis and numerical applications.
\end{abstract}

Keywords: Numerical solution, Schrödinger equation, multistep methods, hybrid methods, P-stability, phase-lag, phase-fitted

PACS: 02.60, 02.70.Bf, 95.10.Ce, 95.10.Eg, 95.75.Pq

\section{Introduction}

The radial Schrödinger equation can be written as:

$$
y^{\prime \prime}(x)=\left[l(l+1) / x^{2}+V(x)-k^{2}\right] y(x) .
$$

Many problems in theoretical physics and chemistry, material sciences, quantum mechanics and quantum chemistry, electronics etc. can be express via the above boundary value problem (see for example [1] $[4])$.

We give the definitions of some terms of (1):

- The function $W(x)=l(l+1) / x^{2}+V(x)$ is called the effective potential. This satisfies $W(x) \rightarrow 0$ as $x \rightarrow \infty$

- The quantity $k^{2}$ is a real number denoting the energy

\footnotetext{
* e-mail: zackanas@uop.gr

$\dagger$ e-mail: dvlachos@uop.gr

$¥$ Highly Cited Researcher, Active Member of the European Academy of Sciences and Arts. Corresponding Member of the European Academy of Sciences Corresponding Member of European Academy of Arts, Sciences and Humanities, Please use the following address for all correspondence: Dr. T.E. Simos, 10 Konitsis Street, Amfithea - Paleon Faliron, GR-175 64 Athens, Greece, Tel: 00302109420 091, e-mail: tsimos.conf@gmail.com, tsimos@mail.ariadne-t.gr
}

(c) 2018 Kluwer Academic Publishers. Printed in the Netherlands. 
- The quantity $l$ is a given integer representing the angular momentum

- $\quad V$ is a given function which denotes the potential.

The boundary conditions are:

$$
y(0)=0
$$

and a second boundary condition, for large values of $x$, determined by physical considerations.

The last years an extended study on the development of numerical methods for the solution of the Schrödinger equation has been done. The aim of this research is the development of fast and reliable methods for the solution of the Schrödinger equation and related problems (see for example [5] - [18], [24] - [127]).

We can divide the numerical methods for the approximate solution of the Schrödinger equation and related problems into two main categories:

1. Methods with constant coefficients

2. Methods with coefficients depending on the frequency of the problem ${ }^{1}$.

The purpose of this paper is to introduce a new methodology for the construction of numerical methods for the approximate solution of the one-dimensional Schrödinger equation and related problems. The new methodology is based on the requirement of vanishing the phase-lag and its derivatives. The efficiency of the new methodology will be studied via the error analysis and the application of the investigated methods to the numerical solution of the radial Schrödinger equation.

More specifically, we will develop a family of hybrid Numerov-type methods of sixth algebraic order. The development of the new family is based on the requirement of vanishing the phase-lag and its derivatives. We will investigate the stability and the error of the methods of the new family. Finally, we will apply both categories of methods the new obtained method to the resonance problem. This is one of the most difficult problems arising from the radial Schrödinger equation. The paper is organized as follows. In Section 2 we present the theory of the new methodology. In section 3 we present the development of the new family of methods. The error analysis is presented in section 4 . In section 5 we will investigate the stability properties of the new

\footnotetext{
1 When using a functional fitting algorithm for the solution of the radial Schrödinger equation, the fitted frequency is equal to: $\sqrt{\left|l(l+1) / x^{2}+V(x)-k^{2}\right|}$
} 
developed methods. In Section 6 the numerical results are presented. Finally, in Section 7 remarks and conclusions are discussed.

\section{Phase-lag analysis of symmetric multistep methods}

For the numerical solution of the initial value problem

$$
y^{\prime \prime}=f(x, y)
$$

consider a multistep method with $m$ steps which can be used over the equally spaced intervals $\left\{x_{i}\right\}_{i=0}^{m} \in[a, b]$ and $h=\left|x_{i+1}-x_{i}\right|, \quad i=$ $0(1) m-1$.

If the method is symmetric then $a_{i}=a_{m-i}$ and $b_{i}=b_{m-i}, \quad i=$ $0(1)\left\lfloor\frac{m}{2}\right\rfloor$.

When a symmetric $2 k$-step method, that is for $i=-k(1) k$, is applied to the scalar test equation

$$
y^{\prime \prime}=-\omega^{2} y
$$

a difference equation of the form

$$
\begin{array}{r}
A_{k}(H) y_{n+k}+\ldots+A_{1}(H) y_{n+1}+A_{0}(H) y_{n}+ \\
+A_{1}(H) y_{n-1}+\ldots+A_{k}(H) y_{n-k}=0
\end{array}
$$

is obtained, where $H=\omega h, h$ is the step length and $A_{0}(H), A_{1}(H), \ldots$, $A_{k}(H)$ are polynomials of $H$.

The characteristic equation associated with (5) is given by:

$$
\begin{array}{r}
A_{k}(H) \lambda^{k}+\ldots+A_{1}(H) \lambda+A_{0}(H)+A_{1}(H) \lambda^{-1}+\ldots \\
+A_{k}(H) \lambda^{-k}=0
\end{array}
$$

THEOREM 1. [102] The symmetric $2 k$-step method with characteristic equation given by (6) has phase-lag order $q$ and phase-lag constant $c$ given by

$$
-c H^{q+2}+O\left(H^{q+4}\right)=\frac{2 A_{k}(H) \cos (k H)+\ldots+2 A_{j}(H) \cos (j H)+\ldots+A_{0}(H)}{2 k^{2} A_{k}(H)+\ldots+2 j^{2} A_{j}(H)+\ldots+2 A_{1}(H)}
$$

The formula proposed from the above theorem gives us a direct method to calculate the phase-lag of any symmetric $2 k$ - step method. 


\section{The New Family of Numerov-Type Hybrid Methods - Construction of the New Methods}

\subsection{First Method of the Family}

We introduce the following family of methods to integrate $y^{\prime \prime}=f(x, y)$ :

$$
\begin{array}{r}
\bar{y}_{n}=y_{n}-a_{0} h^{2}\left(y_{n+1}^{\prime \prime}-2 y_{n}^{\prime \prime}+y_{n-1}^{\prime \prime}\right) \\
y_{n+1}+c_{1} y_{n}+y_{n-1}=h^{2}\left[b_{0}\left(y_{n+1}^{\prime \prime}+y_{n-1}^{\prime \prime}\right)+b_{1} \bar{y}_{n}^{\prime \prime}\right]
\end{array}
$$

The application of the above method to the scalar test equation (4) gives the following difference equation:

$$
A_{1}(H) y_{n+1}+A_{0}(H) y_{n}+A_{1}(H) y_{n-1}=0
$$

where $H=\omega h, h$ is the step length and $A_{0}(H)$ and $A_{1}(H)$ are polynomials of $H$.

The characteristic equation associated with (10) is given by:

$$
A_{1}(H) \lambda+A_{0}(H)+A_{1}(H) \lambda^{-1}=0
$$

where

$$
\begin{array}{r}
A_{1}(H)=1+H^{2} b_{0}+H^{4} b_{1} a_{0} \\
A_{0}(H)=c_{1}+H^{2} b_{1}-2 H^{4} b_{1} a_{0}
\end{array}
$$

By applying $k=1$ in the formula (8), we have that the phase-lag is equal to:

$$
\begin{array}{r}
p h l=\frac{2 A_{1}(H) \cos (H)+A_{0}(H)}{2 A_{1}(H)} \\
=\frac{1}{2} \frac{2\left(1+H^{2} b_{0}+H^{4} b_{1} a_{0}\right) \cos (H)+c_{1}+H^{2} b_{1}-2 H^{4} b_{1} a_{0}}{1+H^{2} b_{0}+H^{4} b_{1} a_{0}}
\end{array}
$$

We demand that the phase-lag is equal to zero and we consider that:

$$
b_{0}=\frac{1}{12}, b_{1}=\frac{5}{6}, c_{1}=-2
$$

Then we find out that:

$$
a_{0}=\frac{-12 \cos (H)-\cos (H) H^{2}+12-5 H^{2}}{10 \cos (H) H^{4}-10 H^{4}}
$$


For small values of $|H|$ the formulae given by (13) are subject to heavy cancellations. In this case the following Taylor series expansions should be used:

$$
\begin{array}{r}
a_{0}=\frac{1}{200}+\frac{1}{5040} H^{2}+\frac{1}{144000} H^{4}+\frac{1}{4435200} H^{6} \\
+\frac{691}{99066240000} H^{8}+\frac{1}{4790016000} H^{10} \\
+\frac{3617}{592812380160000} H^{12}+\frac{43867}{250445794959360000} H^{14} \\
+\frac{174611}{35213055381504000000} H^{16}+\ldots
\end{array}
$$

The behavior of the coefficients is given in the following Figure 1.

The local truncation error of the new proposed method is given by:

$$
\mathrm{LTE}=\frac{h^{8}}{6048}\left(y_{n}^{(8)}+\omega^{2} y_{n}^{(6)}\right)
$$

REMARK 1. The method developed in this section is the same with the obtained by Simos in [116]

\subsection{Second Method of the Family}

Consider the family of methods presented in (9).

The application of the above method to the scalar test equation (4) gives the difference equation (10) and the characteristic equation (10).

By applying $k=1$ in the formula (8), we have that the phase-lag is given by (11). The first derivative of the phase-lag is given by:

$$
\begin{array}{r}
p \dot{h} l=\frac{1}{2} \frac{T_{4}-2 \mathrm{~T}_{0} \sin (H)+2 H b_{1}-8 H^{3} b_{1} a_{0}}{\mathrm{~T}_{0}} \\
-\frac{1}{2} \frac{\left(2 \mathrm{~T}_{0} \cos (H)+c_{1}+H^{2} b_{1}-2 H^{4} b_{1} a_{0}\right)\left(2 H b_{0}+4 H^{3} b_{1} a_{0}\right)}{\mathrm{T}_{0}^{2}} \\
\mathrm{~T}_{0}=1+H^{2} b_{0}+H^{4} b_{1} a_{0} \\
\mathrm{~T}_{4}=2\left(2 H b_{0}+4 H^{3} b_{1} a_{0}\right) \cos (H)
\end{array}
$$

We demand that the phase-lag and its derivative are equal to zero and we consider that:

$$
b_{0}=\frac{1}{12}, b_{1}=\frac{5}{6}
$$




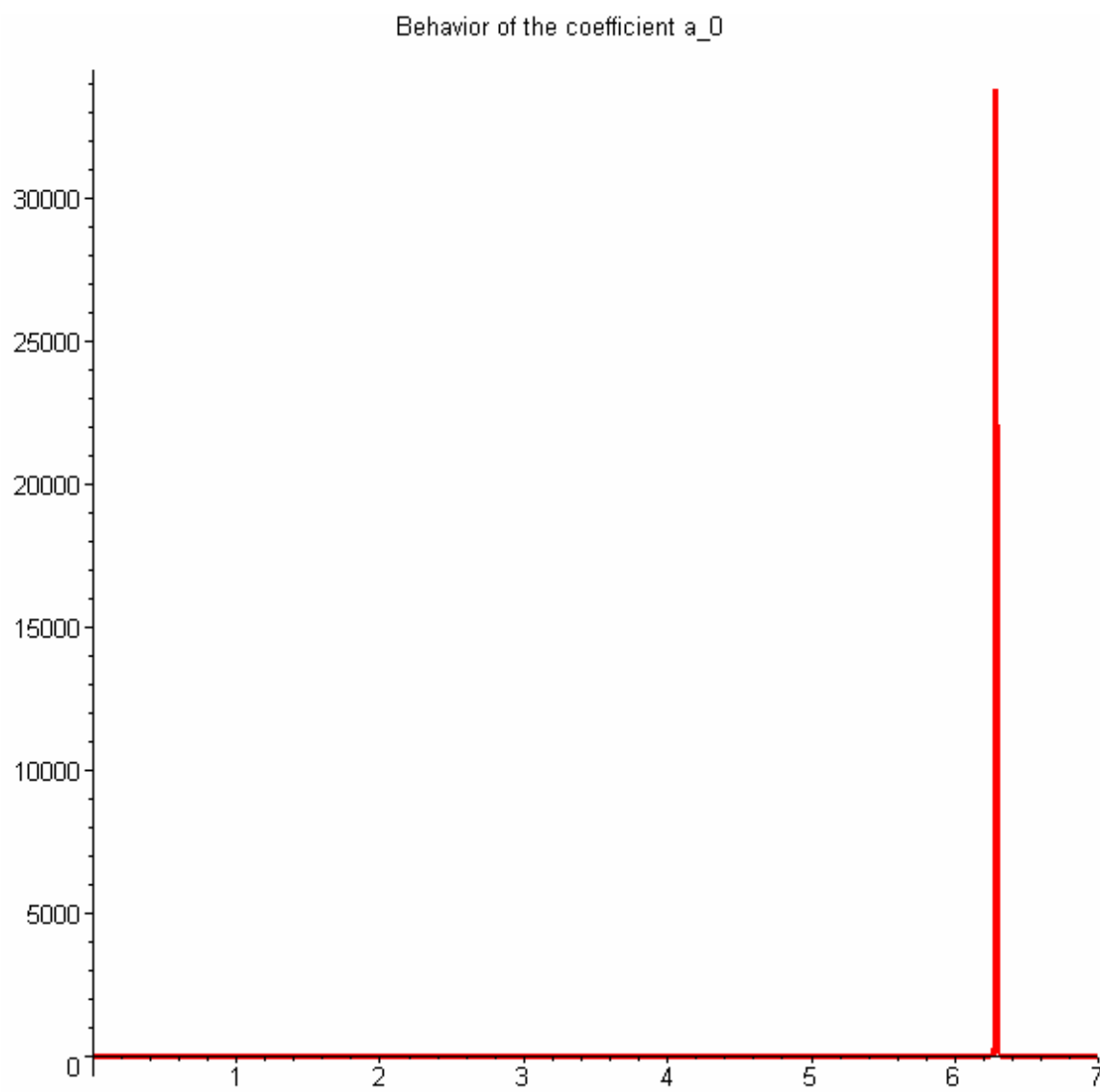

$\mathrm{H}$

Figure 1. Behavior of the coefficient $a_{0}$ of the new method given by (13) for several values of $H$.

Then we find out that:

$$
\begin{array}{r}
a_{0}=\frac{-\sin (H) H^{2}+10 H+2 \cos (H) H-12 \sin (H)}{10 \sin (H) H^{4}-40 \cos (H) H^{3}+40 H^{3}} \\
c_{1}=\left(24 \cos (2 H)+24-48 \cos (H)+H^{2} \cos (2 H)\right. \\
-9 H^{2}+8 \cos (H) H^{2}-6 H^{3} \sin (H) \\
-12 \sin (H) H) /(6 \sin (H) H-24 \cos (H)+24)
\end{array}
$$


For small values of $|H|$ the formulae given by (18) are subject to heavy cancelations. In this case the following Taylor series expansions should be used:

$$
\begin{aligned}
& a_{0}=\frac{1}{200}+\frac{1}{3780} H^{2}+\frac{73}{5443200} H^{4}+\frac{509}{769824000} H^{6} \\
& +\frac{2833543}{88268019840000} H^{8} \\
& +\frac{4912333}{3177648714240000} H^{10} \\
& +\frac{288303913}{3889442026229760000} H^{12}+\frac{165095552521}{46556621053970227200000} H^{14} \\
& +\frac{15619496804053}{92182109686861049856000000} H^{16}+\ldots \\
& c_{1}-2+\frac{1}{18144} H^{8}+\frac{13}{16329600} H^{10}+\frac{31}{461894400} H^{12} \\
& +\frac{308851}{105921623808000} H^{14}+\frac{537907}{3813178457088000} H^{16}+\ldots
\end{aligned}
$$

The behavior of the coefficients is given in the following Figure 2.
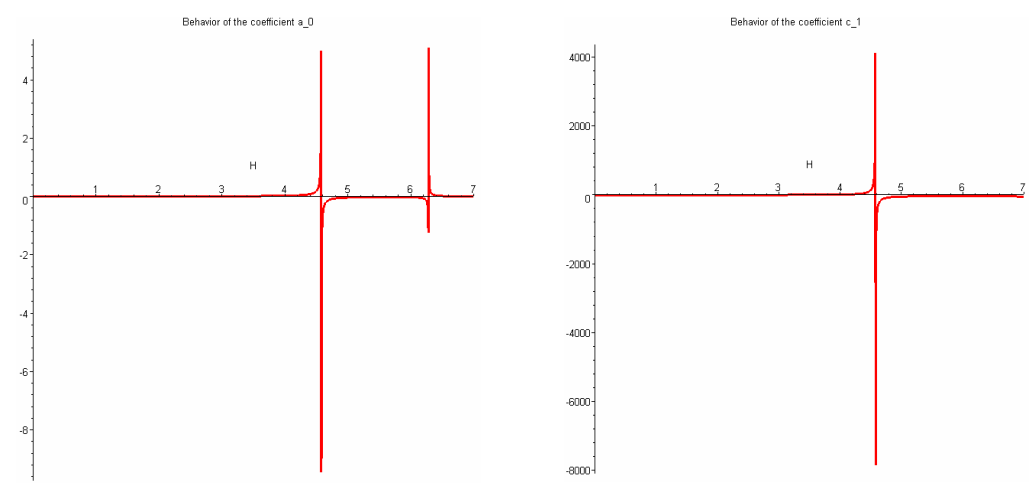

Figure 2. Behavior of the coefficients of the new method given by (18) for several values of $H$.

The local truncation error of the new proposed method is given by:

$$
\mathrm{LTE}=\frac{h^{8}}{18144}\left(3 y_{n}^{(8)}+4 \omega^{2} y_{n}^{(6)}+\omega^{8} y_{n}\right)
$$




\subsection{Third Method of the FAmily}

Consider the family of methods presented in (9).

The application of the above method to the scalar test equation (4) gives the difference equation (10) and the characteristic equation (10).

By applying $k=1$ in the formula (8), we have that the phase-lag is given by (11). The first derivative of the phase-lag is given by (16). The second derivative of the phase-lag can be written as:

$$
\begin{array}{r}
p \ddot{h} l=\frac{1}{2} \frac{T_{3}-4 \mathrm{~T}_{2} \sin (H)-2 \mathrm{~T}_{1} \cos (H)+2 b_{1}-24 b_{1} a_{0} H^{2}}{\mathrm{~T}_{1}} \\
-\frac{\left(2 \mathrm{~T}_{2} \cos (H)-2 \mathrm{~T}_{1} \sin (H)+2 H b_{1}-8 H^{3} b_{1} a_{0}\right) \mathrm{T}_{2}}{\mathrm{~T}_{1}^{2}} \\
+\frac{\left(2 \mathrm{~T}_{1} \cos (H)+c_{1}+H^{2} b_{1}-2 H^{4} b_{1} a_{0}\right) \mathrm{T}_{2}^{2}}{\mathrm{~T}_{1}{ }^{3}} \\
-\frac{1}{2} \frac{\left(2 \mathrm{~T}_{1} \cos (H)+c_{1}+H^{2} b_{1}-2 H^{4} b_{1} a_{0}\right)\left(2 b_{0}+12 b_{1} a_{0} H^{2}\right)}{\mathrm{T}_{1}^{2} \mathrm{~T}_{1}=1+H^{2} b_{0}+H^{4} b_{1} a_{0}} \\
\mathrm{~T}_{2}=2 H b_{0}+4 H^{3} b_{1} a_{0} \\
\mathrm{~T}_{3}=2\left(2 b_{0}+12 b_{1} a_{0} H^{2}\right) \cos (H)
\end{array}
$$

We demand that the phase-lag and its first and second derivative are equal to zero and we consider that:

$$
b_{0}=\frac{1}{12}
$$

Then we find out that: 


$$
\begin{array}{r}
a_{0}=\frac{1}{2}\left(\cos (H) H^{3}+12 \cos (H) H-12 \sin (H)+3 \sin (H) H^{2}\right) / \\
\left(\left(\cos (H)^{2} H^{3}+16 \cos (H)^{2} H+5 \cos (H) H^{2} \sin (H)\right.\right. \\
+72 \cos (H) \sin (H)+2 \cos (H) H^{3}+32 \cos (H) H \\
\left.\left.+2 \sin (H) H^{2}-48 H-2 H^{3}-72 \sin (H)\right) H^{2}\right) \\
c_{1}=\frac{1}{6}\left(24 \cos (H)^{2} H^{2}+\cos (H)^{2} H^{4}+96 \cos (H)^{2}\right. \\
+\cos (H) \sin (H) H^{3}+12 \cos (H) H^{2} \\
-24 \cos (H) \sin (H) H-96 \cos (H)+\cos (H) H^{4} \\
\left.-\sin (H) H^{3}-2 H^{4}-60 \sin (H) H-48 H^{2}\right) / \\
\left(\cos (H) H^{2}+7 \sin (H) H+8-8 \cos (H)\right) \\
b_{1}=-\frac{1}{6}\left(\cos (H)^{2} H^{3}+16 \cos (H)^{2} H+5 \cos (H) H^{2} \sin (H)\right. \\
+72 \cos (H) \sin (H)+2 \cos (H) H^{3} \\
\left.+32 \cos (H) H+2 \sin (H) H^{2}-48 H-2 H^{3}-72 \sin (H)\right) / \\
\left(H\left(\cos (H) H^{2}+7 \sin (H) H+8-8 \cos (H)\right)\right)
\end{array}
$$

For small values of $|H|$ the formulae given by (24) are subject to heavy cancellations. In this case the following Taylor series expansions should be used: 


$$
\begin{array}{r}
a_{0}=\frac{1}{200}+\frac{1}{2520} H^{2}+\frac{31}{907200} H^{4}+\frac{1229}{1197504000} H^{6} \\
+\frac{18427}{980755776000} H^{8}-\frac{669341}{98075577600000} H^{10} \\
-\frac{13764419}{25184162304000000} H^{12}-\frac{281298850211}{5747730994317312000000} H^{14} \\
-\frac{161773544323}{103459157897711616000000} H^{16}+\ldots \\
c_{1}=-2-\frac{1}{6048} H^{8}-\frac{17}{2721600} H^{10}-\frac{43}{57480192} H^{12} \\
-\frac{1515133}{23538138624000} H^{14}-\frac{25819}{4483454976000} H^{16}+\ldots \\
b_{1}=\frac{5}{6}+\frac{1}{3024} H^{6}+\frac{11}{725760} H^{8}+\frac{2353}{1437004800} H^{10} \\
+\frac{186533}{1307674368000} H^{12}+\frac{112457}{8826801984000} H^{14} \\
+\frac{1635421}{1440534083788800} H^{16}+\ldots
\end{array}
$$

The behavior of the coefficients is given in the following Figure 3.

The local truncation error of the new proposed method is given by:

$$
\mathrm{LTE}=\frac{h^{8}}{6048}\left(y_{n}^{(8)}+2 \omega^{2} y_{n}^{(6)}-2 \omega^{6} y_{n}^{(2)}-\omega^{8} y_{n}\right)
$$

\subsection{Fourth Method of the Family}

Consider the family of methods presented in (9).

The application of the above method to the scalar test equation (4) gives the difference equation (10) and the characteristic equation (10).

By applying $k=1$ in the formula (8), we have that the phase-lag is given by (11). The first derivative of the phase-lag is given by (16). The second derivative of the phase-lag is given by (22). The third derivative of the phase-lag can be written as: 


$$
\begin{array}{r}
\dddot{p h l}=\frac{1}{2} \frac{T_{9}-6 \mathrm{~T}_{8} \cos (H)+2 \mathrm{~T}_{5} \sin (H)-48 b_{1} a_{0} H}{\mathrm{~T}_{5}} \\
-\frac{3}{2} \frac{\left(2 \mathrm{~T}_{7} \cos (H)-4 \mathrm{~T}_{8} \sin (H)-2 \mathrm{~T}_{5} \cos (H)+2 b_{1}-24 b_{1} a_{0} H^{2}\right) \mathrm{T}_{8}}{\mathrm{~T}_{5}{ }^{2}} \\
+\frac{3\left(2 \mathrm{~T}_{8} \cos (H)-2 \mathrm{~T}_{5} \sin (H)+2 H b_{1}-8 H^{3} b_{1} a_{0}\right) \mathrm{T}_{8}{ }^{2}}{\mathrm{~T}_{5}{ }^{3}} \\
-\frac{3}{2} \frac{\left(2 \mathrm{~T}_{8} \cos (H)-2 \mathrm{~T}_{5} \sin (H)+2 H b_{1}-8 H^{3} b_{1} a_{0}\right) \mathrm{T}_{7}}{\mathrm{~T}_{5}{ }^{2}-\frac{3 \mathrm{~T}_{6} \mathrm{~T}_{8}{ }^{3}}{\mathrm{~T}_{5}{ }^{4}}} \\
+\frac{3 \mathrm{~T}_{6} \mathrm{~T}_{8} \mathrm{~T}_{7}}{\mathrm{~T}_{5}{ }^{3}}-\frac{12 \mathrm{~T}_{6} b_{1} a_{0} H}{\mathrm{~T}_{5}{ }^{2}} \\
\mathrm{~T}_{5}=1+H^{2} b_{0}+H^{4} b_{1} a_{0} \\
\mathrm{~T}_{6}=2 \mathrm{~T}_{5} \cos (H)+c_{1}+H^{2} b_{1}-2 H^{4} b_{1} a_{0} \\
\mathrm{~T}_{7}=2 b_{0}+12 b_{1} a_{0} H^{2} \\
\mathrm{~T}_{8}=2 H b_{0}+4 H^{3} b_{1} a_{0} \\
\mathrm{~T}_{9}=48 b_{1} a_{0} H \cos (H)-6 \mathrm{~T}_{7} \sin (H(27)
\end{array}
$$

We demand that the phase-lag and its first, second and third derivative are equal to zero and we find out that: 


$$
\begin{aligned}
& a_{0}=\frac{1}{4}\left(3 \cos (H)^{2}+\cos (H)^{2} H^{2}+2 H^{2}-3\right) \\
& /\left(\left(6 \cos (H)^{3} H+6 \sin (H) \cos (H)^{2}\right.\right. \\
& -2 \cos (H)^{2} H^{2} \sin (H)+\cos (H)^{2} H^{3} \\
& +3 \cos (H)^{2} H-6 \cos (H) \sin (H) \\
& -4 \cos (H) H^{2} \sin (H)-12 \cos (H) H+2 H^{3} \\
& \left.\left.+3 H+12 \sin (H) H^{2}\right) H\right) \\
& c_{1}=-2\left(-12 \cos (H)^{3} H+\cos (H)^{2} H^{3}-21 \cos (H)^{2} H\right. \\
& -12 \sin (H) \cos (H)^{2}-4 \cos (H)^{2} H^{2} \sin (H)+12 \cos (H) \sin (H) \\
& \left.-8 \cos (H) H^{2} \sin (H)+24 \cos (H) H+2 H^{3}+9 H+24 \sin (H) H^{2}\right) / \\
& \left(\cos (H)^{2} H^{3}-21 \cos (H)^{2} H+8 \cos (H) H^{2} \sin (H)\right. \\
& -12 \cos (H) H-12 \cos (H) \sin (H)+4 \sin (H) H^{2} \\
& \left.+33 H+12 \sin (H)+2 H^{3}\right) \\
& b_{0}=-2\left(3 \cos (H)^{2} H+\cos (H)^{2} H^{3}+6 \cos (H) \sin (H)\right. \\
& +4 \cos (H) H^{2} \sin (H)+6 \cos (H) H \\
& \left.+2 \sin (H) H^{2}-9 H-6 \sin (H)+2 H^{3}\right) / \\
& \left(\left(\cos (H)^{2} H^{3}-21 \cos (H)^{2} H+8 \cos (H) H^{2} \sin (H)\right.\right. \\
& -12 \cos (H) H-12 \cos (H) \sin (H) \\
& \left.\left.+4 \sin (H) H^{2}+33 H+12 \sin (H)+2 H^{3}\right) H^{2}\right) \\
& b_{1}=4\left(6 \cos (H)^{3} H+6 \sin (H) \cos (H)^{2}-2 \cos (H)^{2} H^{2} \sin (H)\right. \\
& +\cos (H)^{2} H^{3}+3 \cos (H)^{2} H-6 \cos (H) \sin (H) \\
& \left.-4 \cos (H) H^{2} \sin (H)-12 \cos (H) H+2 H^{3}+3 H+12 \sin (H) H^{2}\right) / \\
& \left(\left(\cos (H)^{2} H^{3}-21 \cos (H)^{2} H+8 \cos (H) H^{2} \sin (H)-12 \cos (H) H\right.\right. \\
& \left.-12 \cos (H) \sin (H)+4 \sin (H) H^{2}+33 H+12 \sin (H)+2 H^{3}\right) H^{2}(28)
\end{aligned}
$$

For small values of $|H|$ the formulae given by (28) are subject to heavy cancellations. In this case the following Taylor series expansions should be used: 


$$
\begin{aligned}
& a_{0}=\frac{1}{200}+\frac{1}{1260} H^{2}+\frac{29}{504000} H^{4}+\frac{1433}{1164240000} H^{6} \\
& -\frac{63101}{363242880000} H^{8}-\frac{2228861}{127135008000000} H^{10} \\
& -\frac{8804897}{77806624896000000} H^{12}+\frac{240953700049}{2048959660011264000000} H^{14} \\
& +\frac{9699610781879}{819583864004505600000000} H^{16}+\ldots \\
& c_{1}=-2+\frac{1}{6048} H^{8}+\frac{1}{43200} H^{10}+\frac{1}{532224} H^{12} \\
& +\frac{41}{5943974400} H^{14}-\frac{601}{24141680640} H^{16}+\ldots \\
& b_{0}=\frac{1}{12}-\frac{1}{1008} H^{4}-\frac{31}{181440} H^{6} \\
& -\frac{221}{13685760} H^{8}-\frac{619}{1345344000} H^{10} \\
& +\frac{25031}{174356582400} H^{12}+\frac{84256583}{2667655710720000} H^{14} \\
& +\frac{1030007057}{290289444157440000} H^{16}+\ldots \\
& b_{1}=\frac{5}{6}+\frac{1}{504} H^{4}-\frac{29}{90720} H^{6} \\
& -\frac{3271}{47900160} H^{8}-\frac{35293}{4540536000} H^{10} \\
& -\frac{36019}{87178291200} H^{12}+\frac{47333617}{1333827855360000} H^{14} \\
& +\frac{294008389}{24562952967168000} H^{16}+\ldots
\end{aligned}
$$

The behavior of the coefficients is given in the following Figure 4.

The local truncation error of the new proposed method is given by:

$$
\mathrm{LTE}=\frac{h^{8}}{6048}\left(y_{n}^{(8)}+4 \omega^{2} y_{n}^{(6)}+6 \omega^{4} y_{n}^{(4)}+4 \omega^{6} y_{n}^{(2)}+\omega^{8} y_{n}\right)
$$

\section{Error Analysis}

We will study the following methods: 

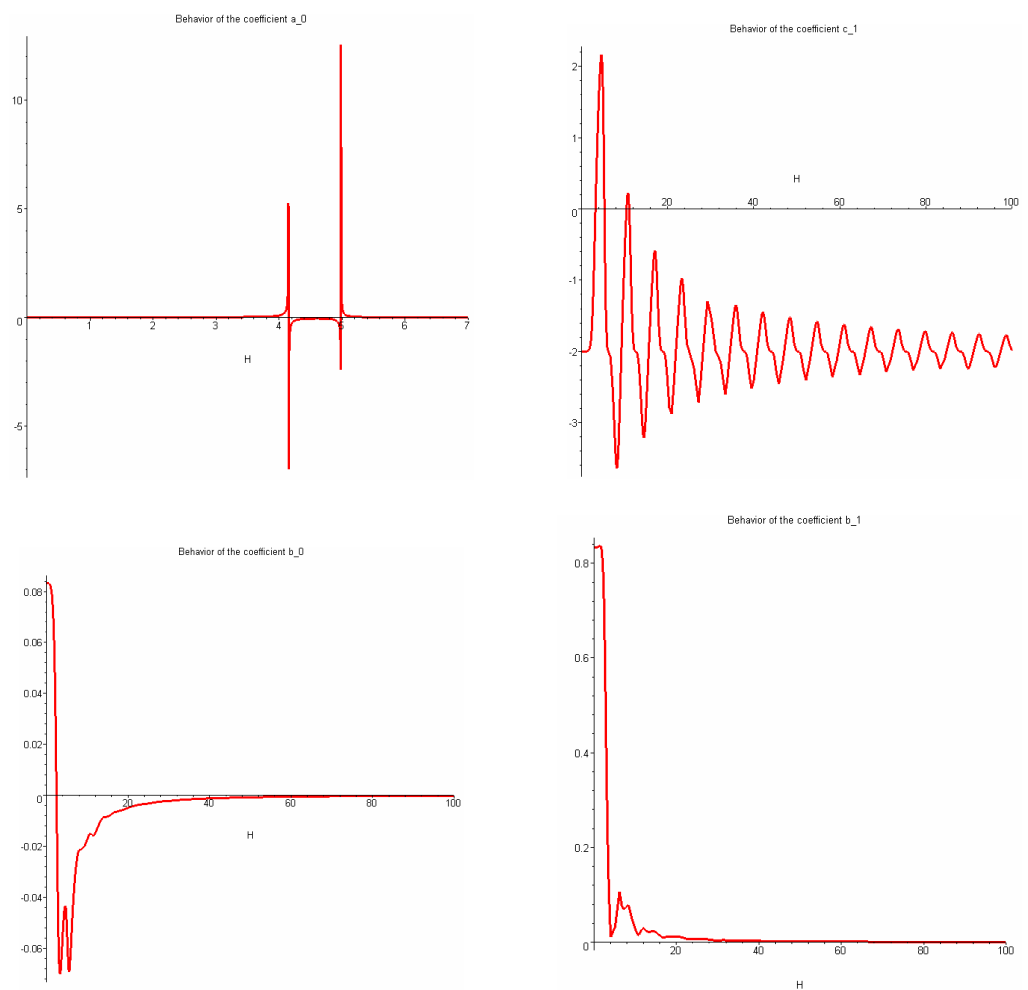

Figure 4. Behavior of the coefficients of the new method given by (28) for several values of $H$.

- The First Method of the Family (mentioned as PL1)

- The Second Method of the Family (mentioned as PL2)

- The Third Method of the Family (mentioned as PL3)

- The Fourth Method of the Family (mentioned as PL4)

The error analysis is based on the following steps:

- The radial time independent Schrödinger equation is of the form

$$
y^{\prime \prime}(x)=f(x) y(x)
$$

- Based on the paper of Ixaru and Rizea [25], the function $\mathrm{f}(\mathrm{x})$ can be written in the form:

$$
f(x)=g(x)+G
$$


where $g(x)=V(x)-V_{c}=g$, where $V_{c}$ is the constant approximation of the potential and $G=v^{2}=V_{c}-E$.

- We express the derivatives $y_{n}^{(i)}, i=2,3,4, \ldots$, which are terms of the local truncation error formulae, in terms of the equation (31). The expressions are presented as polynomials of $G$.

- Finally, we substitute the expressions of the derivatives, produced in the previous step, into the local truncation error formulae.

Based on the procedure mentioned above and on the formulae:

$$
\begin{array}{r}
y_{n}^{(2)}=\left(\mathrm{V}(x)-V_{c}+G\right) \mathrm{y}(x) \\
y_{n}^{(4)}=\left(\frac{d^{2}}{d x^{2}} \mathrm{~V}(x)\right) \mathrm{y}(x)+2\left(\frac{d}{d x} \mathrm{~V}(x)\right)\left(\frac{d}{d x} \mathrm{y}(x)\right) \\
+\left(\mathrm{V}(x)-V_{c}+G\right)\left(\frac{d^{2}}{d x^{2}} \mathrm{y}(x)\right) \\
y_{n}^{(6)}=\left(\frac{d^{4}}{d x^{4}} \mathrm{~V}(x)\right) \mathrm{y}(x)+4\left(\frac{d^{3}}{d x^{3}} \mathrm{~V}(x)\right)\left(\frac{d}{d x} \mathrm{y}(x)\right) \\
+3\left(\frac{d^{2}}{d x^{2}} \mathrm{~V}(x)\right)\left(\frac{d^{2}}{d x^{2}} \mathrm{y}(x)\right) \\
+4\left(\frac{d}{d x} \mathrm{~V}(x)\right)^{2} \mathrm{y}(x) \\
+6\left(\mathrm{~V}(x)-V_{c}+G\right)\left(\frac{d}{d x} \mathrm{y}(x)\right)\left(\frac{d}{d x} \mathrm{~V}(x)\right) \\
+4\left(\mathrm{U}(x)-V_{c}+G\right) \mathrm{y}(x)\left(\frac{d^{2}}{d x^{2}} \mathrm{~V}(x)\right) \\
+\left(\mathrm{V}(x)-V_{c}+G\right)^{2}\left(\frac{d^{2}}{d x^{2}} \mathrm{y}(x)\right) \ldots
\end{array}
$$

we obtain the following expressions: 


\section{The First Method of the FAmily}

$$
\begin{aligned}
& \operatorname{LTE}_{\mathrm{PL} 1}=h^{8}\left[-\frac{1}{6048} \mathrm{~g}(x) \mathrm{y}(x) G^{3}+\left(-\frac{5}{2016}\left(\frac{d^{2}}{d x^{2}} \mathrm{~g}(x)\right) \mathrm{y}(x)\right.\right. \\
& \left.-\frac{1}{1008}\left(\frac{d}{d x} \mathrm{~g}(x)\right)\left(\frac{d}{d x} \mathrm{y}(x)\right)-\frac{1}{2016} \mathrm{~g}(x)^{2} \mathrm{y}(x)\right) G^{2}+( \\
& -\frac{5}{2016}\left(\frac{d^{4}}{d x^{4}} \mathrm{~g}(x)\right) \mathrm{y}(x)-\frac{5}{1512}\left(\frac{d^{3}}{d x^{3}} \mathrm{~g}(x)\right)\left(\frac{d}{d x} \mathrm{y}(x)\right) \\
& -\frac{1}{336} \mathrm{~g}(x)\left(\frac{d}{d x} \mathrm{y}(x)\right)\left(\frac{d}{d x} \mathrm{~g}(x)\right)-\frac{37}{6048} \mathrm{~g}(x) \mathrm{y}(x)\left(\frac{d^{2}}{d x^{2}} \mathrm{~g}(x)\right) \\
& \left.-\frac{1}{252}\left(\frac{d}{d x} \mathrm{~g}(x)\right)^{2} \mathrm{y}(x)-\frac{1}{2016} \mathrm{~g}(x)^{3} \mathrm{y}(x)\right) G \\
& -\frac{1}{6048}\left(\frac{d^{6}}{d x^{6}} \mathrm{~g}(x)\right) \mathrm{y}(x)-\frac{1}{1008}\left(\frac{d^{5}}{d x^{5}} \mathrm{~g}(x)\right)\left(\frac{d}{d x} \mathrm{y}(x)\right) \\
& -\frac{1}{378} \mathrm{~g}(x) \mathrm{y}(x)\left(\frac{d^{4}}{d x^{4}} \mathrm{~g}(x)\right)-\frac{5}{2016}\left(\frac{d^{2}}{d x^{2}} \mathrm{~g}(x)\right)^{2} \mathrm{y}(x) \\
& -\frac{13}{3024}\left(\frac{d}{d x} \mathrm{~g}(x)\right) \mathrm{y}(x)\left(\frac{d^{3}}{d x^{3}} \mathrm{~g}(x)\right)-\frac{1}{252} \mathrm{~g}(x)\left(\frac{d}{d x} \mathrm{y}(x)\right)\left(\frac{d^{3}}{d x^{3}} \mathrm{~g}(x)\right) \\
& -\frac{1}{504} \mathrm{~g}(x)^{2}\left(\frac{d}{d x} \mathrm{y}(x)\right)\left(\frac{d}{d x} \mathrm{~g}(x)\right) \\
& -\frac{1}{126}\left(\frac{d}{d x} \mathrm{~g}(x)\right)\left(\frac{d}{d x} \mathrm{y}(x)\right)\left(\frac{d^{2}}{d x^{2}} \mathrm{~g}(x)\right) \\
& -\frac{11}{3024} \mathrm{~g}(x)^{2} \mathrm{y}(x)\left(\frac{d^{2}}{d x^{2}} \mathrm{~g}(x)\right)-\frac{1}{216} \mathrm{~g}(x) \mathrm{y}(x)\left(\frac{d}{d x} \mathrm{~g}(x)\right)^{2} \\
& \left.-\frac{1}{6048} \mathrm{~g}(x)^{4} \mathrm{y}(x)\right]
\end{aligned}
$$


The Second Method of the Family

$$
\begin{array}{r}
\text { LTE }_{\text {PL } 2}=h^{8}\left[\left(\frac{19}{9072}\left(\frac{d^{2}}{d x^{2}} \mathrm{~g}(x)\right) \mathrm{y}(x)+\frac{1}{1512}\left(\frac{d}{d x} \mathrm{~g}(x)\right)\left(\frac{d}{d x} \mathrm{y}(x)\right)\right.\right. \\
\left.+\frac{1}{3024} \mathrm{~g}(x)^{2} \mathrm{y}(x)\right) G^{2}+\left(\frac{11}{4536}\left(\frac{d^{4}}{d x^{4}} \mathrm{~g}(x)\right) \mathrm{y}(x)\right. \\
+\frac{1}{324}\left(\frac{d^{3}}{d x^{3}} \mathrm{~g}(x)\right)\left(\frac{d}{d x} \mathrm{y}(x)\right)+\frac{1}{378} \mathrm{~g}(x)\left(\frac{d}{d x} \mathrm{y}(x)\right)\left(\frac{d}{d x} \mathrm{~g}(x)\right) \\
+\frac{13}{2268} \mathrm{~g}(x) \mathrm{y}(x)\left(\frac{d^{2}}{d x^{2}} \mathrm{~g}(x)\right)+\frac{17}{4536}\left(\frac{d}{d x} \mathrm{~g}(x)\right)^{2} \mathrm{y}(x) \\
\left.+\frac{1}{2268} \mathrm{~g}(x)^{3} \mathrm{y}(x)\right) G+\frac{1}{6048}\left(\frac{d^{6}}{d x^{6}} \mathrm{~g}(x)\right) \mathrm{y}(x) \\
+\frac{1}{1008}\left(\frac{d^{5}}{d x^{5}} \mathrm{~g}(x)\right)\left(\frac{d}{d x} \mathrm{y}(x)\right)+\frac{1}{378} \mathrm{~g}(x) \mathrm{y}(x)\left(\frac{d^{4}}{d x^{4}} \mathrm{~g}(x)\right) \\
+\frac{5}{2016}\left(\frac{d^{2}}{d x^{2}} \mathrm{~g}(x)\right)^{2} \mathrm{y}(x)+\frac{13}{3024}\left(\frac{d}{d x} \mathrm{~g}(x)\right) \mathrm{y}(x)\left(\frac{d^{3}}{d x^{3}} \mathrm{~g}(x)\right) \\
+\frac{1}{252} \mathrm{~g}(x)\left(\frac{d}{d x} \mathrm{y}(x)\right)\left(\frac{d^{3}}{d x^{3}} \mathrm{~g}(x)\right)+\frac{1}{504} \mathrm{~g}(x)^{2}\left(\frac{d}{d x} \mathrm{y}(x)\right)\left(\frac{d}{d x} \mathrm{~g}(x)\right) \\
\mathrm{g}(x))\left(\frac{d}{d x} \mathrm{y}(x)\right)\left(\frac{d^{2}}{d x^{2}} \mathrm{~g}(x)\right)+\frac{11}{3024} \mathrm{~g}(x)^{2} \mathrm{y}(x)\left(\frac{d^{2}}{d x^{2}} \mathrm{~g}(x)\right) \\
\left.+\frac{1}{216} \mathrm{~g}(x) \mathrm{y}(x)\left(\frac{d}{d x} \mathrm{~g}(x)\right)^{2}+\frac{1}{6048} \mathrm{~g}(x)^{4} \mathrm{y}(x)\right](34)
\end{array}
$$




\section{The Third Method of the Family}

$$
\begin{array}{r}
\text { LTE }_{\text {P } 3}=h^{8}\left[\frac{1}{756}\left(\frac{d^{2}}{d x^{2}} \mathrm{~g}(x)\right) \mathrm{y}(x) G^{2}\right. \\
+\left(\frac{1}{432}\left(\frac{d^{4}}{d x^{4}} \mathrm{~g}(x)\right) \mathrm{y}(x)+\frac{1}{378}\left(\frac{d^{3}}{d x^{3}} \mathrm{~g}(x)\right)\left(\frac{d}{d x} \mathrm{y}(x)\right)\right. \\
+\frac{1}{504} \mathrm{~g}(x)\left(\frac{d}{d x} \mathrm{y}(x)\right)\left(\frac{d}{d x} \mathrm{~g}(x)\right) \\
+\frac{5}{1008} \mathrm{~g}(x) \mathrm{y}(x)\left(\frac{d^{2}}{d x^{2}} \mathrm{~g}(x)\right)+\frac{5}{1512}\left(\frac{d}{d x} \mathrm{~g}(x)\right)^{2} \mathrm{y}(x) \\
\left.+\frac{1}{3024} \mathrm{~g}(x)^{3} \mathrm{y}(x)\right) G+\frac{1}{6048}\left(\frac{d^{6}}{d x^{6}} \mathrm{~g}(x)\right) \mathrm{y}(x) \\
+\frac{1}{1008}\left(\frac{d^{5}}{d x^{5}} \mathrm{~g}(x)\right)\left(\frac{d}{d x} \mathrm{y}(x)\right) \\
+\frac{1}{378} \mathrm{~g}(x) \mathrm{y}(x)\left(\frac{d^{4}}{d x^{4}} \mathrm{~g}(x)\right) \\
\left.+\frac{13}{2016}\left(\frac{d^{2}}{d x^{2}} \mathrm{~g}(x)\right)^{2} \mathrm{y}(x)+\frac{d}{3024} \mathrm{~g}(x)\right) \mathrm{y}(x)\left(\frac{d^{3}}{d x^{3}} \mathrm{~g}(x)\right) \\
+\frac{1}{504} \mathrm{~g}(x)^{2}\left(\frac{d}{d x} \mathrm{y}(x)\right)\left(\frac{d^{3}}{d x^{3}} \mathrm{~g}(x)\right) \\
+\frac{1}{d 26} \mathrm{~g}\left(\frac{d}{d x} \mathrm{~g}(x)\right)\left(\frac{d}{d x} \mathrm{y}(x)\right)\left(\frac{d^{2}}{d x^{2}} \mathrm{~g}(x)\right)+\frac{11}{3024} \mathrm{~g}(x)^{2} \mathrm{y}(x)\left(\frac{d^{2}}{d x^{2}} \mathrm{~g}(x)\right) \\
\left.+\frac{1}{216} \mathrm{~g}(x) \mathrm{y}(x)\left(\frac{d}{d x} \mathrm{~g}(x)\right)^{2}+\frac{1}{6048} \mathrm{~g}(x)^{4} \mathrm{y}(x)\right](35)
\end{array}
$$


The Fourth Method of the Family

$$
\begin{array}{r}
\text { LTE }_{\text {PL4 }}= \\
h^{8}\left[\left(\frac{1}{504}\left(\frac{d^{4}}{d x^{4}} \mathrm{~g}(x)\right) \mathrm{y}(x)+\frac{1}{756}\left(\frac{d^{3}}{d x^{3}} \mathrm{~g}(x)\right)\left(\frac{d}{d x} \mathrm{y}(x)\right)\right.\right. \\
\left.+\frac{1}{378} \mathrm{~g}(x) \mathrm{y}(x)\left(\frac{d^{2}}{d x^{2}} \mathrm{~g}(x)\right)+\frac{1}{504}\left(\frac{d}{d x} \mathrm{~g}(x)\right)^{2} \mathrm{y}(x)\right) G \\
+\frac{1}{6048}\left(\frac{d^{6}}{d x^{6}} \mathrm{~g}(x)\right) \mathrm{y}(x)+\frac{1}{1008}\left(\frac{d^{5}}{d x^{5}} \mathrm{~g}(x)\right)\left(\frac{d}{d x} \mathrm{y}(x)\right) \\
+\frac{1}{378} \mathrm{~g}(x) \mathrm{y}(x)\left(\frac{d^{4}}{d x^{4}} \mathrm{~g}(x)\right)+\frac{5}{2016}\left(\frac{d^{2}}{d x^{2}} \mathrm{~g}(x)\right)^{2} \mathrm{y}(x) \\
+\frac{13}{3024}\left(\frac{d}{d x} \mathrm{~g}(x)\right) \mathrm{y}(x)\left(\frac{d^{3}}{d x^{3}} \mathrm{~g}(x)\right) \\
+\frac{1}{252} \mathrm{~g}(x)\left(\frac{d}{d x} \mathrm{y}(x)\right)\left(\frac{d^{3}}{d x^{3}} \mathrm{~g}(x)\right)+\frac{1}{504} \mathrm{~g}(x)^{2}\left(\frac{d}{d x} \mathrm{y}(x)\right)\left(\frac{d}{d x} \mathrm{~g}(x)\right) \\
+\frac{1}{126}\left(\frac{d}{d x} \mathrm{~g}(x)\right)\left(\frac{d}{d x} \mathrm{y}(x)\right)\left(\frac{d^{2}}{d x^{2}} \mathrm{~g}(x)\right) \\
+\frac{11}{3024} \mathrm{~g}(x)^{2} \mathrm{y}(x)\left(\frac{d^{2}}{d x^{2}} \mathrm{~g}(x)\right) \\
\left.+\frac{1}{216} \mathrm{~g}(x) \mathrm{y}(x)\left(\frac{d}{d x} \mathrm{~g}(x)\right)^{2}+\frac{1}{6048} \mathrm{~g}(x)^{4} \mathrm{y}(x)\right]
\end{array}
$$

We consider two cases in terms of the value of $E$ :

- The Energy is close to the potential, i.e. $G=V_{c}-E \approx 0$. So only the free terms of the polynomials in $G$ are considered. Thus for these values of $G$, the methods are of comparable accuracy. This is because the free terms of the polynomials in $G$, are the same for the cases of the classical method and of the new developed methods.

- $G \gg 0$ or $G \ll 0$. Then $|G|$ is a large number. So, we have the following asymptotic expansions of the equations (33), (34), (35) and (36).

The First Method of the Family

$$
\operatorname{LTE}_{\mathrm{PL} 1}=h^{8}\left(-\frac{1}{6048} \mathrm{~g}(x) \mathrm{y}(x) G^{3}+\ldots\right)
$$


The Second Method of the Family

$$
\begin{aligned}
& \operatorname{LTE}_{\text {PL2 }}=h^{8}\left(\frac{19}{9072}\left(\frac{d^{2}}{d x^{2}} \mathrm{~g}(x)\right) \mathrm{y}(x)+\frac{1}{1512}\left(\frac{d}{d x} \mathrm{~g}(x)\right)\left(\frac{d}{d x} \mathrm{y}(x)\right)\right. \\
& \left.\left.+\frac{1}{3024} \mathrm{~g}(x)^{2} \mathrm{y}(x)\right) G^{2}+\ldots\right)
\end{aligned}
$$

The Third Method of the Family

$$
\operatorname{LTE}_{\mathrm{PL} 3}=h^{8}\left(\frac{1}{756}\left(\frac{d^{2}}{d x^{2}} \mathrm{~g}(x)\right) \mathrm{y}(x) G^{2}+\ldots\right)
$$

The Fourth Method of the Family

$$
\begin{aligned}
& \text { LTE }_{\text {PL }}=h^{8}\left(\left(\frac{1}{504}\left(\frac{d^{4}}{d x^{4}} \mathrm{~g}(x)\right) \mathrm{y}(x)+\frac{1}{756}\left(\frac{d^{3}}{d x^{3}} \mathrm{~g}(x)\right)\left(\frac{d}{d x} \mathrm{y}(x)\right)\right.\right. \\
&\left.\left.+\frac{1}{378} \mathrm{~g}(x) \mathrm{y}(x)\left(\frac{d^{2}}{d x^{2}} \mathrm{~g}(x)\right)+\frac{1}{504}\left(\frac{d}{d x} \mathrm{~g}(x)\right)^{2} \mathrm{y}(x)\right) G+\ldots\right)(39)
\end{aligned}
$$

From the above equations we have the following theorem:

THEOREM 2. For the First Method of the New Family of Methods the error increases as the third power of G. For the Second and Third Methods of the New Family of Methods the error increases as the second power of G. For the Fourth Method of the New Family of Methods the error increases as the first power of $G$. It is easy one to see that the coefficient of the second power of $G$ in the case of the second method of the New Family of Methods is 1.583333333 times larger than the coefficient of the second power of $G$ in the case of the third method of the New Family of Methods. So, for the numerical solution of the time independent radial Schrödinger equation the new obtained Fourth Method of the New Family of Methods is the most accurate one, especially for large values of $|G|=\left|V_{c}-E\right|$.

\section{Stability Analysis}

We apply the new family of methods to the scalar test equation:

$$
y^{\prime \prime}=-t^{2} y
$$


where $t \neq \omega$. We obtain the following difference equation:

$$
A_{1}(H, s) y_{n+1}+A_{0}(H, s) y_{n}+A_{1}(H, s) y_{n-1}=0
$$

where $s=t h, h$ is the step length and $A_{0}(H, s)$ and $A_{1}(H, s)$ are polynomials of $s$.

The characteristic equation associated with (41) is given by:

$$
A_{1}(H, s) s+A_{0}(H, s)+A_{1}(H, s) s^{-1}=0
$$

where

$$
\begin{array}{r}
A_{1}(H, s)=1+s^{2} b_{0}+s^{4} b_{1} a_{0} \\
A_{0}(H, s)=c_{1}+s^{2} b_{1}-2 s^{4} b_{1} a_{0}
\end{array}
$$

DEFINITION 1. (see [19]) A symmetric four-step method with the characteristic equation given by (41) is said to have an interval of periodicity $\left(0, w_{0}^{2}\right)$ if, for all $w \in\left(0, w_{0}^{2}\right)$, the roots $z_{i}, i=1,2$ satisfy

$$
z_{1,2}=e^{ \pm i \theta(t h)},\left|z_{i}\right| \leq 1, i=3,4
$$

where $\theta(t h)$ is a real function of $t h$ and $s=t h$.

DEFINITION 2. (see [19]) A method is called P-stable if its interval of periodicity is equal to $(0, \infty)$.

THEOREM 3. (see [20]) A symmetric two-step method with the characteristic equation given by (41) is said to have a nonzero interval of periodicity $\left(0, s_{0}^{2}\right)$ if, for all $s \in\left(0, s_{0}^{2}\right)$ the following relations are hold

$$
P_{1}(H, s)>0, P_{2}(H, s)>0,
$$

where $H=\omega h, s=t h$ and:

$$
\begin{gathered}
P_{1}(H, s)=A_{0}(H, s)+2 A_{1}(H, s)>0, \\
P_{2}(H, s)=A_{0}(H, s)-2 A_{1}(H, s)>0,
\end{gathered}
$$

DEFINITION 3. A method is called singularly almost P-stable if its interval of periodicity is equal to $(0, \infty)-S^{2}$ only when the frequency of the phase fitting is the same as the frequency of the scalar test equation, i.e. $H=s$.

\footnotetext{
${ }^{2}$ where $S$ is a set of distinct points
} 
Based on (42) the stability polynomials (45) for the new developed methods take the form:

$$
\begin{array}{r}
P_{1}(H, s)=c_{1}+v^{2} b_{1}+2+2 v^{2} b_{0}, \\
P_{2}(H, s)=c_{1}+v^{2} b_{1}-4 v^{4} b_{1} a_{0}-2-2 v^{2} b_{0}
\end{array}
$$

In Figures 5, 6, 7 and 8 we present the $s-H$ planes for the methods developed in this paper. A shadowed area denotes the $s-H$ region where the method is unstable, while a white area denotes the region where the method is stable. In Figure 5 we present the $s-H$ plane for the first method of the new family of method developed in this paper (paragraph 3.1). In Figure 6 we present the $s-H$ plane for the second method of the new family of method developed in this paper (paragraph 3.2). In Figure 7 we present the $s-H$ plane for the third method of the new family of method developed in this paper (paragraph 3.3). Finally, in Figure 8 we present the $s-H$ plane for the fourth method of the new family of method developed in this paper (paragraph 3.4).

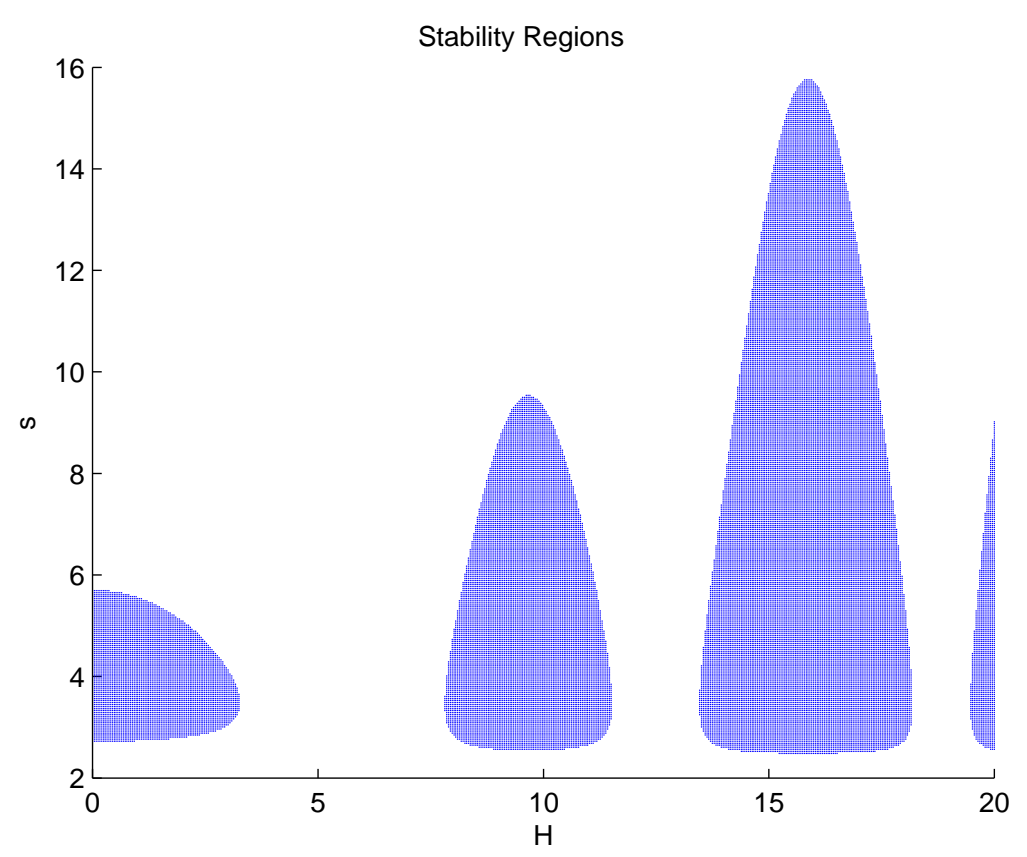

Figure 5. $s-H$ plane of the first method of the new family of method developed in this paper (paragraph 3.1) 


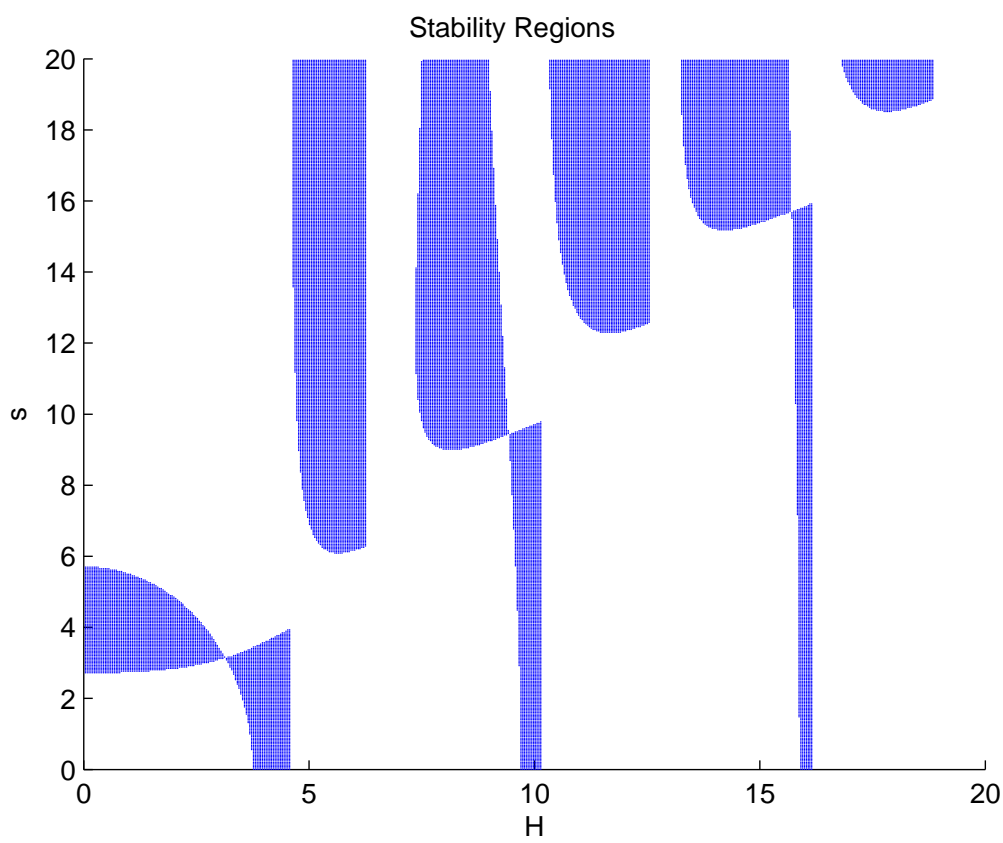

Figure 6. $s-H$ plane of the second method of the new family of method developed in this paper (paragraph 3.2)

In the case that the frequency of the scalar test equation is equal with the frequency of phase fitting, i.e. in the case that $H=s$, we have the following figure for the stability polynomials of the new developed methods. A method is P-stable if the $s-H$ plane is not shadowed. From the above diagrams it is easy for one to see that the interval of periodicity of all the new methods is equal to: $\left(0, \pi^{2}\right)$.

REMARK 2. For the solution of the Schrödinger equation the frequency of the exponential fitting is equal to the frequency of the scalar test equation. So, it is necessary to observe the surroundings of the first diagonal of the $w-H$ plane. 


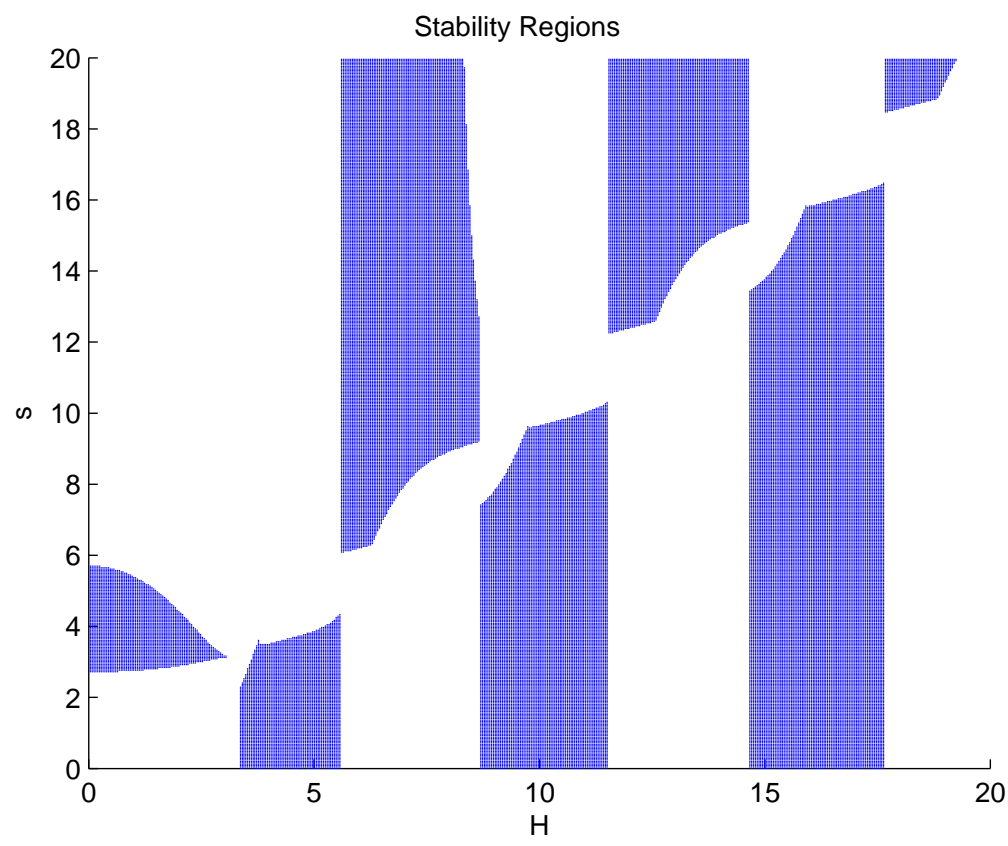

Figure 7. $s-H$ plane of the third method of the new family of method developed in this paper (paragraph 3.3)

\section{Numerical results - Conclusion}

In order to illustrate the efficiency of the new methods obtained in paragraphs $3.1-3.4$, we apply them to the radial time independent Schrödinger equation.

In order to apply the new methods to the radial Schrödinger equation the value of parameter $v$ is needed. For every problem of the one-dimensional Schrödinger equation given by (1) the parameter $v$ is given by

$$
v=\sqrt{|q(x)|}=\sqrt{|V(x)-E|}
$$

where $V(x)$ is the potential and $E$ is the energy.

\subsection{WoOdS-SAXON POTENTIAL}

We use the well known Woods-Saxon potential given by

$$
V(x)=\frac{u_{0}}{1+z}-\frac{u_{0} z}{a(1+z)^{2}}
$$

with $z=\exp \left[\left(x-X_{0}\right) / a\right], u_{0}=-50, a=0.6$, and $X_{0}=7.0$. 


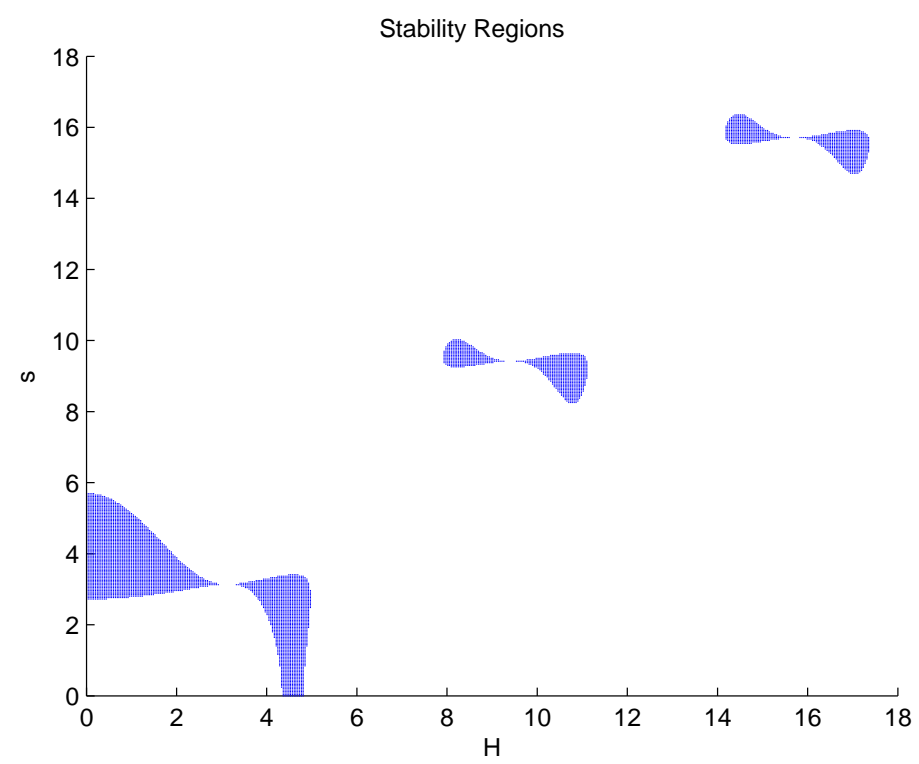

Figure 8. $s-H$ plane of the fourth method of the new family of method developed in this paper (paragraph 3.4)
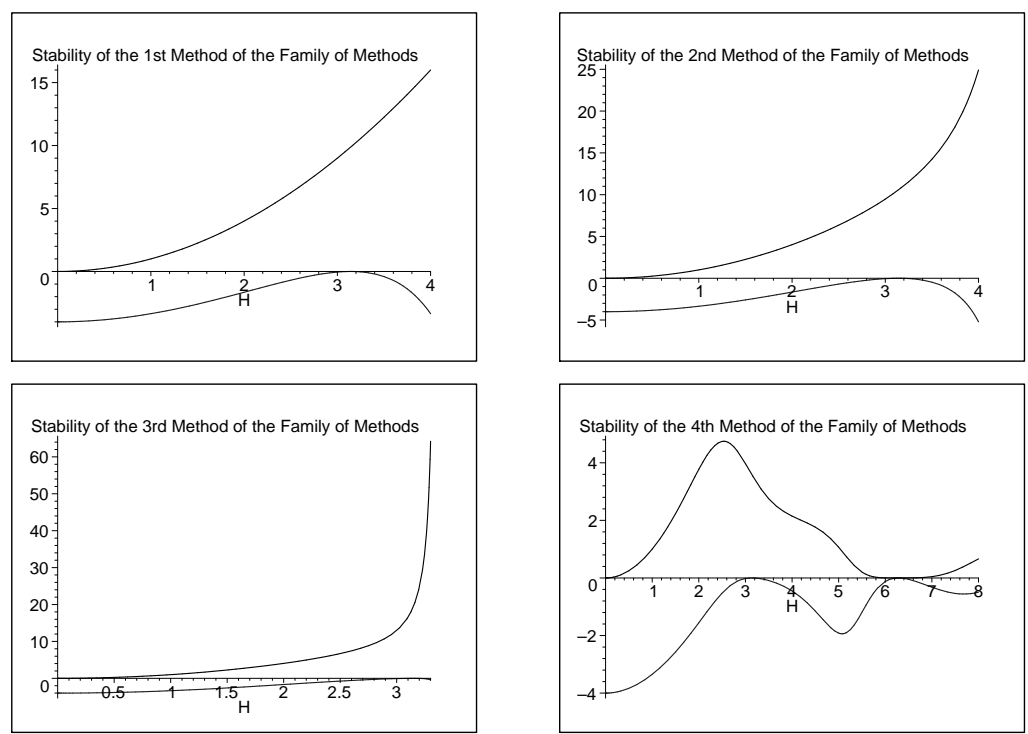

Figure 9. Stability polynomials of the new developed methods in the case that $H=s$

The behavior of Woods-Saxon potential is shown in the Figure 10. 

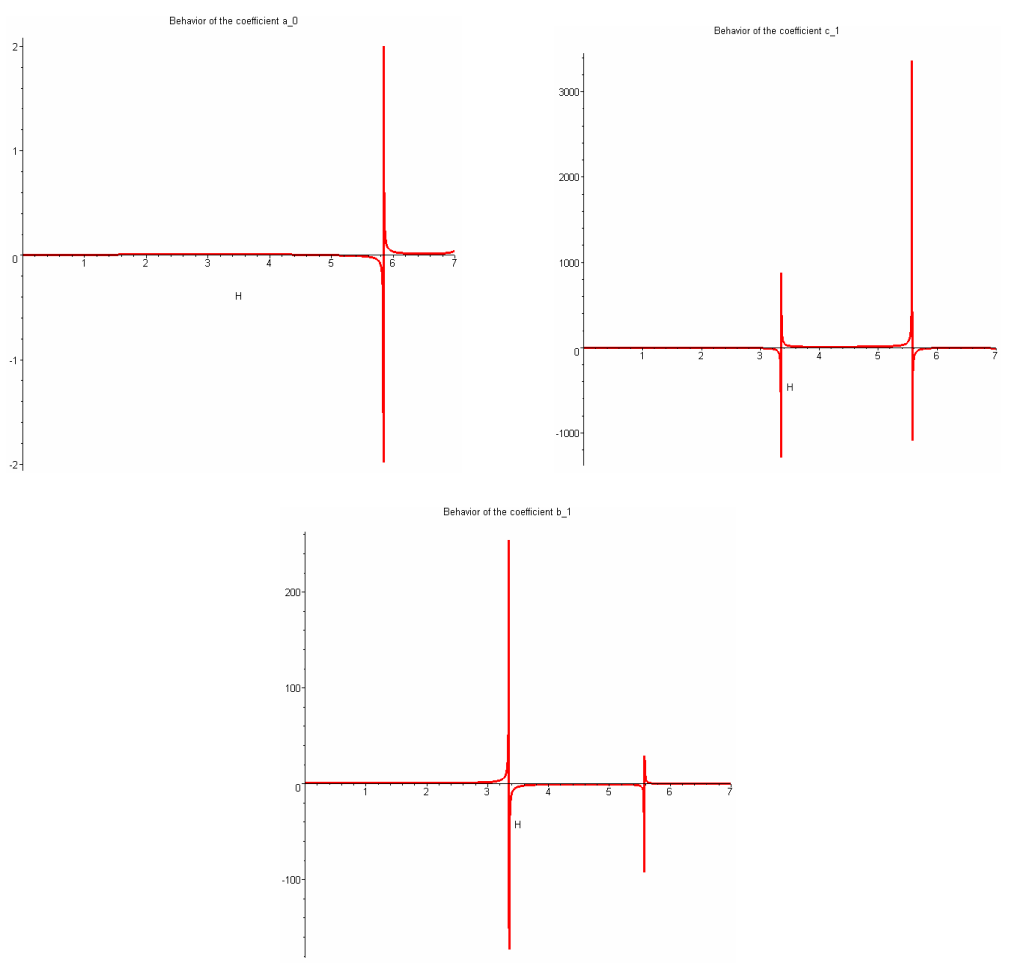

Figure 10. Behavior of the coefficients of the new method given by (24) for several values of $H$.

It is well known that for some potentials, such as the Woods-Saxon potential, the definition of parameter $v$ is not given as a function of $x$ but it is based on some critical points which have been defined from the investigation of the appropriate potential (see for details [13]).

For the purpose of obtaining our numerical results it is appropriate to choose $v$ as follows (see for details [13]):

$$
v=\left\{\begin{array}{cl}
\sqrt{-50+E}, & \text { for } \mathrm{x} \in[0,6.5-2 \mathrm{~h}] \\
\sqrt{-37.5+E}, & \text { for } \mathrm{x}=6.5-h \\
\sqrt{-25+E}, & \text { for } \mathrm{x}=6.5 \\
\sqrt{-12.5+E}, & \text { for } \mathrm{x}=6.5+h \\
\sqrt{E}, & \text { for } \mathrm{x} \in[6.5+2 \mathrm{~h}, 15]
\end{array}\right.
$$




\subsection{Radial Schrödinger Equation - The Resonance PROBLEM}

Consider the numerical solution of the radial time independent Schrödinger equation (1) in the well-known case of the Woods-Saxon potential (48). In order to solve this problem numerically we need to approximate the true (infinite) interval of integration by a finite interval. For the purpose of our numerical illustration we take the domain of integration as $x \in[0,15]$. We consider equation (1) in a rather large domain of energies, i.e. $E \in[1,1000]$.

In the case of positive energies, $E=k^{2}$, the potential dies away faster than the term $\frac{l(l+1)}{x^{2}}$ and the Schrödinger equation effectively reduces to

$$
y^{\prime \prime}(x)+\left(k^{2}-\frac{l(l+1)}{x^{2}}\right) y(x)=0
$$

for $x$ greater than some value $X$.

The above equation has linearly independent solutions $k x j_{l}(k x)$ and $k x n_{l}(k x)$ where $j_{l}(k x)$ and $n_{l}(k x)$ are the spherical Bessel and Neumann functions respectively. Thus the solution of equation (1) (when $x \rightarrow \infty$ ) has the asymptotic form

$$
\begin{array}{r}
y(x) \simeq A k x j_{l}(k x)-B k x n_{l}(k x) \\
\simeq A C\left[\sin \left(k x-\frac{l \pi}{2}\right)+\tan \delta_{l} \cos \left(k x-\frac{l \pi}{2}\right)\right]
\end{array}
$$

where $\delta_{l}$ is the phase shift, that is calculated from the formula

$$
\tan \delta_{l}=\frac{y\left(x_{2}\right) S\left(x_{1}\right)-y\left(x_{1}\right) S\left(x_{2}\right)}{y\left(x_{1}\right) C\left(x_{1}\right)-y\left(x_{2}\right) C\left(x_{2}\right)}
$$

for $x_{1}$ and $x_{2}$ distinct points in the asymptotic region (we choose $x_{1}$ as the right hand end point of the interval of integration and $x_{2}=$ $\left.x_{1}-h\right)$ with $S(x)=k x j_{l}(k x)$ and $C(x)=-k x n_{l}(k x)$. Since the problem is treated as an initial-value problem, we need $y_{0}$ before starting a one-step method. From the initial condition we obtain $y_{0}$. With these starting values we evaluate at $x_{1}$ of the asymptotic region the phase shift $\delta_{l}$.

For positive energies we have the so-called resonance problem. This problem consists either of finding the phase-shift $\delta_{l}$ or finding those $E$, for $E \in[1,1000]$, at which $\delta_{l}=\frac{\pi}{2}$. We actually solve the latter problem, known as the resonance problem when the positive eigenenergies lie under the potential barrier.

The boundary conditions for this problem are:

$$
y(0)=0, y(x)=\cos (\sqrt{E} x) \text { for large } x .
$$




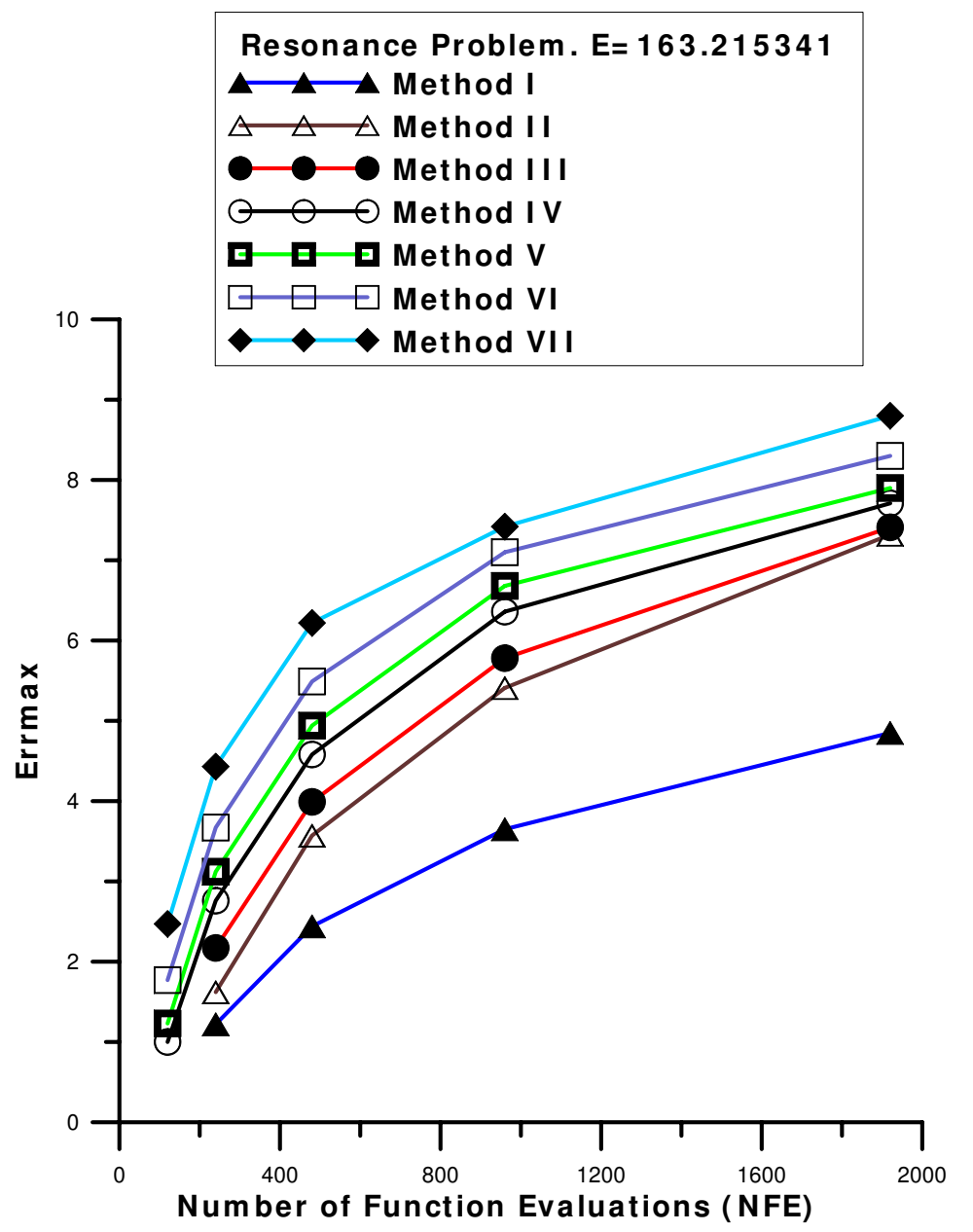

Figure 11. Error Errmax for several values of $\mathrm{n}$ for the eigenvalue $E_{1}=163.215341$. The nonexistence of a value of Errmax indicates that for this value of n, Errmax is positive

We compute the approximate positive eigenenergies of the WoodsSaxon resonance problem using:

- The Numerov's method which is indicated as Method I

- The Exponentially-fitted four-step method developed by Raptis [16] which is indicated as Method II 


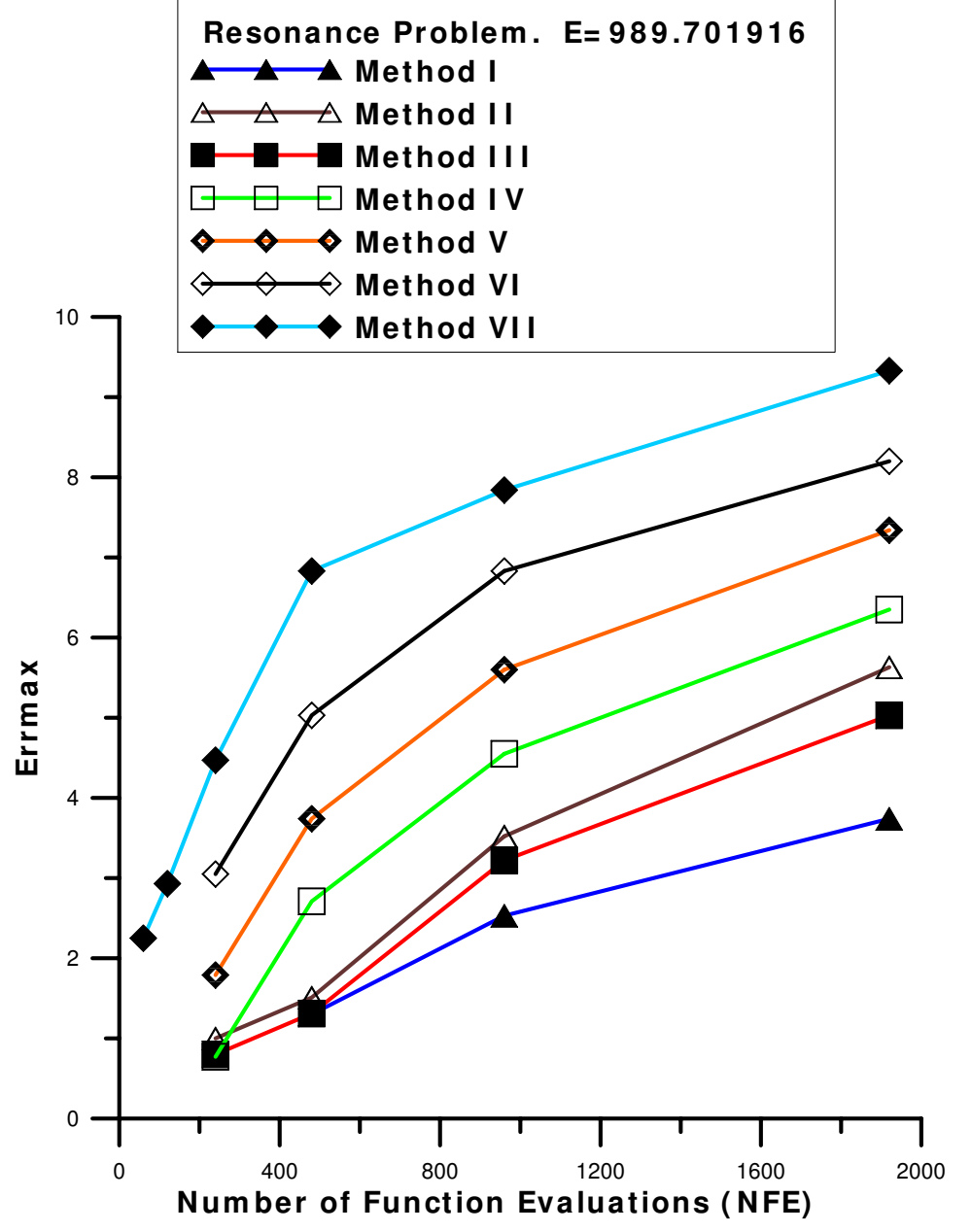

Figure 12. Error Errmax for several values of $\mathrm{n}$ for the eigenvalue $E_{3}=989.701916$. The nonexistence of a value of Errmax indicates that for this value of n, Errmax is positive

- The Two-Step Numerov-type Method with minimum phase-lag produced by Chawla and Rao [23] which is indicated as Method III

- The new Two-Step Numerov-Type Method with phase-lag equal to zero obtained in paragraph 3.1 which is indicated as Method IV. 
- The new Two-Step Numerov-Type Method with phase-lag and its first derivative equal to zero obtained in paragraph 3.2 which is indicated as Method V.

- The new Two-Step Numerov-Type Method with phase-lag and its first and second derivatives equal to zero obtained in paragraph 3.3 which is indicated as Method VI.

- The new Two-Step Numerov-Type Method with phase-lag and its first, second and third derivatives equal to zero obtained in paragraph 3.4 which is indicated as Method VII.

The computed eigenenergies are compared with exact ones. In Figure 11 we present the maximum absolute error $\log _{10}(E r r)$ where

$$
\text { Err }=\left|E_{\text {calculated }}-E_{\text {accurate }}\right|
$$

of the eigenenergy $E_{1}$, for several values of NFE $=$ Number of Function Evaluations. In Figure 12 we present the maximum absolute error $\log _{10}(E r r)$ where

$$
\text { Err }=\left|E_{\text {calculated }}-E_{\text {accurate }}\right|
$$

of the eigenenergy $E_{3}$, for several values of NFE = Number of Function Evaluations.

\section{Conclusions}

In the present paper we have developed a family of methods of sixth algebraic order for the numerical solution of the radial Schrödinger equation.

More specifically we have developed:

1. A Two-Step Numerov-Type Method with phase-lag equal to zero

2. A Two-Step Numerov-Type Method with phase-lag and its first derivative equal to zero

3. A Two-Step Numerov-Type Method with phase-lag and its first and second derivatives equal to zero

4. A Two-Step Numerov-Type Method with phase-lag and its first, second and third derivatives equal to zero 
We have applied the new method to the resonance problem of the radial Schrödinger equation.

Based on the results presented above we have the following conclusions:

- The Exponentially-fitted four-step method developed by Raptis [16] (denoted as Method II) is more efficient than the Numerov's Method (denoted Method I).

- The Two-Step Numerov-type Method with minimum phase-lag produced by Chawla and Rao [23] (Method III) is more efficient than the Exponentially-fitted four-step method developed by Raptis [16] (Method II) for the energy 163.215341 and less efficient for the energy 989.701916.

- The new developed methods are much more efficient than the older ones.

- The Two-Step Numerov-Type Method with phase-lag and its first derivative equal to zero (Method V) is more efficient than the New Two-Step Numerov-Type Method with phase-lag equal to zero (Method IV)

- The Two-Step Numerov-Type Method with phase-lag and its first and second derivatives equal to zero (Method VI) is more efficient than the Two-Step Numerov-Type Method with phase-lag and its first derivative equal to zero (Method V)

- The Two-Step Numerov-Type Method with phase-lag and its first, second and third derivatives equal to zero (Method VII) is more efficient than the Two-Step Numerov-Type Method with phase-lag and its first and second derivatives equal to zero (Method VI)

All computations were carried out on a IBM PC-AT compatible 80486 using double precision arithmetic with 16 significant digits accuracy (IEEE standard).

\section{References}

1. L.Gr. Ixaru and M. Micu, Topics in Theoretical Physics. Central Institute of Physics, Bucharest, 1978.

2. L.D. Landau and F.M. Lifshitz: Quantum Mechanics. Pergamon, New York, 1965.

3. I. Prigogine, Stuart Rice (Eds): Advances in Chemical Physics Vol. 93: New Methods in Computational Quantum Mechanics, John Wiley \& Sons, 1997. 
4. G. Herzberg, Spectra of Diatomic Molecules, Van Nostrand, Toronto, 1950.

5. T.E. Simos, Atomic Structure Computations in Chemical Modelling: Applications and Theory (Editor: A. Hinchliffe, UMIST), The Royal Society of Chemistry 38-142(2000).

6. T.E. Simos, Numerical methods for 1D, 2D and 3D differential equations arising in chemical problems, Chemical Modelling: Application and Theory, The Royal Society of Chemistry, 2(2002),170-270.

7. T.E. Simos and P.S. Williams, On finite difference methods for the solution of the Schrödinger equation, Computers \&3 Chemistry 23 513-554(1999).

8. T.E. Simos: Numerical Solution of Ordinary Differential Equations with Periodical Solution. Doctoral Dissertation, National Technical University of Athens, Greece, 1990 (in Greek).

9. A. Konguetsof and T.E. Simos, On the Construction of exponentially-fitted methods for the numerical solution of the Schrödinger Equation, Journal of Computational Methods in Sciences and Engineering 1 143-165(2001).

10. A.D. Raptis and A.C. Allison: Exponential - fitting methods for the numerical solution of the Schrödinger equation, Computer Physics Communications, 14 1-5(1978).

11. A.D. Raptis, Exponential multistep methods for ordinary differential equations, Bull. Greek Math. Soc. 25 113-126(1984).

12. L.Gr. Ixaru, Numerical Methods for Differential Equations and Applications, Reidel, Dordrecht - Boston - Lancaster, 1984.

13. L.Gr. Ixaru and M. Rizea, A Numerov-like scheme for the numerical solution of the Schrödinger equation in the deep continuum spectrum of energies. Comput. Phys. Commun. 19 23-27(1980).

14. T. E. Simos, P. S. Williams: A New Runge-Kutta-Nystrom Method with Phase-Lag of Order Infinity for the Numerical Solution of the Schrödinger Equation, MATCH Commun. Math. Comput. Chem. 45 123-137(2002).

15. T. E. Simos, Multiderivative Methods for the Numerical Solution of the Schrödinger Equation, MATCH Commun. Math. Comput. Chem. 45 726(2004).

16. A.D. Raptis, Exponentially-fitted solutions of the eigenvalue Shrödinger equation with automatic error control, Computer Physics Communications, 28 427-431(1983)

17. A.D. Raptis, On the numerical solution of the Schrodinger equation, Computer Physics Communications, 24 1-4(1981)

18. Zacharoula Kalogiratou and T.E. Simos, A P-stable exponentially-fitted method for the numerical integration of the Schrödinger equation, Applied Mathematics and Computation, 112 99-112(2000).

19. J.D. Lambert and I.A. Watson, Symmetric multistep methods for periodic initial values problems, J. Inst. Math. Appl. 18 189-202(1976).

20. A.D. Raptis and T.E. Simos, A four-step phase-fitted method for the numerical integration of second order initial-value problem, BIT, 31 160-168(1991).

21. Peter Henrici, Discrete variable methods in ordinary differential equations, John Wiley \& Sons, 1962.

22. M.M. Chawla, Uncoditionally stable Noumerov-type methods for second order differential equations, BIT, 23 541-542(1983).

23. M. M. Chawla and P. S. Rao, A Noumerov-type method with minimal phaselag for the integration of second order periodic initial-value problems, Journal of Computational and Applied Mathematics 11(3) 277-281(1984) 
24. Liviu Gr. Ixaru and Guido Vanden Berghe, Exponential Fitting, Series on Mathematics and its Applications, Vol. 568, Kluwer Academic Publisher, The Netherlands, 2004.

25. L. Gr. Ixaru and M. Rizea, Comparison of some four-step methods for the numerical solution of the Schrödinger equation, Computer Physics Communications, 38(3) 329-337(1985)

26. Z.A. Anastassi, T.E. Simos, A family of exponentially-fitted Runge-Kutta methods with exponential order up to three for the numerical solution of the Schrödinger equation, J. Math. Chem 41 (1) 79-100 (2007)

27. T. Monovasilis, Z. Kalogiratou , T.E. Simos, Trigonometrically fitted and exponentially fitted symplectic methods for the numerical integration of the Schrödinger equation, J. Math. Chem 40 (3) 257-267 (2006)

28. G. Psihoyios, T.E. Simos, The numerical solution of the radial Schrödinger equation via a trigonometrically fitted family of seventh algebraic order Predictor-Corrector methods, J. Math. Chem 40 (3) 269-293 (2006)

29. T.E. Simos, A four-step exponentially fitted method for the numerical solution of the Schrödinger equation, J. Math. Chem 40 (3) 305-318 (2006)

30. T. Monovasilis, Z. Kalogiratou, T.E. Simos, Exponentially fitted symplectic methods for the numerical integration of the Schrödinger equation J. Math. Chem 37 (3) 263-270 (2005)

31. Z. Kalogiratou, T. Monovasilis, T.E. Simos, Numerical solution of the two-dimensional time independent Schrödinger equation with Numerov-type methods J. Math. Chem 37 (3) 271-279 (2005)

32. Z.A. Anastassi, T.E. Simos, Trigonometrically fitted Runge-Kutta methods for the numerical solution of the Schrödinger equation J. Math. Chem 37 (3) 281-293 (2005)

33. G. Psihoyios, T.E. Simos, Sixth algebraic order trigonometrically fitted predictor-corrector methods for the numerical solution of the radial Schrödinger equation, J. Math. Chem 37 (3) 295-316 (2005)

34. D.P. Sakas, T.E. Simos, A family of multiderivative methods for the numerical solution of the Schrödinger equation, J. Math. Chem 37 (3) 317-331 (2005)

35. T.E. Simos, Exponentially - fitted multiderivative methods for the numerical solution of the Schrödinger equation, J. Math. Chem 36 (1) 13-27 (2004)

36. K. Tselios, T.E. Simos, Symplectic methods of fifth order for the numerical solution of the radial Shrodinger equation, J. Math. Chem 35 (1) 55-63 (2004)

37. T.E. Simos, A family of trigonometrically-fitted symmetric methods for the efficient solution of the Schrödinger equation and related problems J. Math. Chem 34 (1-2) 39-58 JUL 2003

38. K. Tselios, T.E. Simos, Symplectic methods for the numerical solution of the radial Shrödinger equation, J. Math. Chem 34 (1-2) 83-94 (2003)

39. J. Vigo-Aguiar J, T.E. Simos, Family of twelve steps exponential fitting symmetric multistep methods for the numerical solution of the Schrödinger equation, J. Math. Chem 32 (3) 257-270 (2002)

40. G. Avdelas, E. Kefalidis, T.E. Simos, New P-stable eighth algebraic order exponentially-fitted methods for the numerical integration of the Schrödinger equation, J. Math. Chem 31 (4) 371-404 (2002) 
41. T.E. Simos, J. Vigo-Aguiar, Symmetric eighth algebraic order methods with minimal phase-lag for the numerical solution of the Schrödinger equation $J$. Math. Chem 31 (2) 135-144 (2002)

42. Z. Kalogiratou , T.E. Simos, Construction of trigonometrically and exponentially fitted Runge-Kutta-Nystrom methods for the numerical solution of the Schrödinger equation and related problems a method of 8th algebraic order, J. Math. Chem 31 (2) 211-232

43. T.E. Simos, J. Vigo-Aguiar, A modified phase-fitted Runge-Kutta method for the numerical solution of the Schrödinger equation, J. Math. Chem 30 (1) 121-131 (2001)

44. G. Avdelas, A. Konguetsof, T.E. Simos, A generator and an optimized generator of high-order hybrid explicit methods for the numerical solution of the Schrödinger equation. Part 1. Development of the basic method, J. Math. Chem 29 (4) 281-291 (2001)

45. G. Avdelas, A. Konguetsof, T.E. Simos, A generator and an optimized generator of high-order hybrid explicit methods for the numerical solution of the Schrödinger equation. Part 2. Development of the generator; optimization of the generator and numerical results, J. Math. Chem 29 (4) 293-305 (2001)

46. J. Vigo-Aguiar, T.E. Simos, A family of P-stable eighth algebraic order methods with exponential fitting facilities, J. Math. Chem 29 (3) 177-189 (2001)

47. T.E. Simos, A new explicit Bessel and Neumann fitted eighth algebraic order method for the numerical solution of the Schrödinger equation J. Math. Chem 27 (4) 343-356 (2000)

48. G. Avdelas, T.E. Simos, Embedded eighth order methods for the numerical solution of the Schrödinger equation, J. Math. Chem 26 (4) 327-341 1999,

49. T.E. Simos, A family of P-stable exponentially-fitted methods for the numerical solution of the Schrödinger equation, J. Math. Chem 25 (1) 65-84 (1999)

50. T.E. Simos, Some embedded modified Runge-Kutta methods for the numerical solution of some specific Schrödinger equations, J. Math. Chem 24 (1-3) 23-37 (1998)

51. T.E. Simos, Eighth order methods with minimal phase-lag for accurate computations for the elastic scattering phase-shift problem, J. Math. Chem 21 (4) 359-372 (1997)

52. P. Amodio, I. Gladwell and G. Romanazzi, Numerical Solution of General Bordered ABD Linear Systems by Cyclic Reduction, JNAIAM J. Numer. Anal. Indust. Appl. Math 1(1) 5-12(2006)

53. S. D. Capper, J. R. Cash and D. R. Moore, Lobatto-Obrechkoff Formulae for 2nd Order Two-Point Boundary Value Problems, JNAIAM J. Numer. Anal. Indust. Appl. Math 1(1) 13-25 (2006)

54. S. D. Capper and D. R. Moore, On High Order MIRK Schemes and HermiteBirkhoff Interpolants, JNAIAM J. Numer. Anal. Indust. Appl. Math 1(1) 27-47 (2006)

55. J. R. Cash, N. Sumarti, T. J. Abdulla and I. Vieira, The Derivation of Interpolants for Nonlinear Two-Point Boundary Value Problems, JNAIAM J. Numer. Anal. Indust. Appl. Math 1(1) 49-58 (2006)

56. J. R. Cash and S. Girdlestone, Variable Step Runge-Kutta-Nystrm Methods for the Numerical Solution of Reversible Systems, JNAIAM J. Numer. Anal. Indust. Appl. Math 1(1) 59-80 (2006) 
57. Jeff R. Cash and Francesca Mazzia, Hybrid Mesh Selection Algorithms Based on Conditioning for Two-Point Boundary Value Problems, JNAIAM J. Numer. Anal. Indust. Appl. Math 1(1) 81-90 (2006)

58. Felice Iavernaro, Francesca Mazzia and Donato Trigiante, Stability and Conditioning in Numerical Analysis, JNAIAM J. Numer. Anal. Indust. Appl. Math 1(1) 91-112 (2006)

59. Felice Iavernaro and Donato Trigiante, Discrete Conservative Vector Fields Induced by the Trapezoidal Method, JNAIAM J. Numer. Anal. Indust. Appl. Math 1(1) 113-130 (2006)

60. Francesca Mazzia, Alessandra Sestini and Donato Trigiante, BS Linear Multistep Methods on Non-uniform Meshes, JNAIAM J. Numer. Anal. Indust. Appl. Math 1(1) 131-144 (2006)

61. L.F. Shampine, P.H. Muir, H. Xu, A User-Friendly Fortran BVP Solver, JNAIAM J. Numer. Anal. Indust. Appl. Math 1(2) 201-217 (2006)

62. G. Vanden Berghe and M. Van Daele, Exponentially- fitted Strmer/Verlet methods, JNAIAM J. Numer. Anal. Indust. Appl. Math 1(3) 241-255 (2006)

63. L. Aceto, R. Pandolfi, D. Trigiante, Stability Analysis of Linear Multistep Methods via Polynomial Type Variation, JNAIAM J. Numer. Anal. Indust. Appl. Math 2(1-2) 1-9 (2007)

64. G. Psihoyios, A Block Implicit Advanced Step-point (BIAS) Algorithm for Stiff Differential Systems, Computing Letters 2(1-2) 51-58(2006)

65. W.H. Enright, On the use of 'arc length' and 'defect' for mesh selection for differential equations, Computing Letters 1(2) 47-52(2005)

66. T.E. Simos, P-stable Four-Step Exponentially-Fitted Method for the $\mathrm{Nu}-$ merical Integration of the Schrödinger Equation, Computing Letter 1(1) 37-45(2005).

67. T.E. Simos, Stabilization of a Four-Step Exponentially-Fitted Method and its Application to the Schrödinger Equation, International Journal of Modern Physics C 18(3) 315-328(2007).

68. Zhongcheng Wang, P-stable linear symmetric multistep methods for periodic initial-value problems, Computer Physics Communications 171 162174(2005)

69. T.E. Simos, A Runge-Kutta Fehlberg method with phase-lag of order infinity for initial value problems with oscillating solution, Computers and Mathematics with Applications 25 95-101(1993).

70. T.E. Simos, Runge-Kutta interpolants with minimal phase-lag, Computers and Mathematics with Applications 26 43-49(1993).

71. T.E. Simos, Runge-Kutta-Nyström interpolants for the numerical integration of special second-order periodic initial-value problems, Computers and Mathematics with Applications 26 7-15(1993).

72. T.E. Simos and G.V. Mitsou, A family of four-step exponential fitted methods for the numerical integration of the radial Schrödinger equation, Computers and Mathematics with Applications 28 41-50(1994).

73. T.E. Simos and G. Mousadis, A two-step method for the numerical solution of the radial Schrdinger equation, Computers and Mathematics with Applications 29 31-37(1995).

74. G. Avdelas and T.E. Simos, Block Runge-Kutta methods for periodic initial-value problems, Computers and Mathematics with Applications 3169 83(1996).

75. G. Avdelas and T.E. Simos, Embedded methods for the numerical solution of the Schrödinger equation, Computers and Mathematics with Applications 31 85-102(1996). 
76. G. Papakaliatakis and T.E. Simos, A new method for the numerical solution of fourth order BVPs with oscillating solutions, Computers and Mathematics with Applications 32 1-6(1996).

77. T.E. Simos, An extended Numerov-type method for the numerical solution of the Schrödinger equation, Computers and Mathematics with Applications 33 67-78(1997).

78. T.E. Simos, A new hybrid imbedded variable-step procedure for the numerical integration of the Schrödinger equation, Computers and Mathematics with Applications 36 51-63(1998).

79. T.E. Simos, Bessel and Neumann Fitted Methods for the Numerical Solution of the Schrödinger equation, Computers $\&$ Mathematics with Applications 42 833-847(2001).

80. A. Konguetsof and T.E. Simos, An exponentially-fitted and trigonometricallyfitted method for the numerical solution of periodic initial-value problems, Computers and Mathematics with Applications 45 547-554(2003).

81. Z.A. Anastassi and T.E. Simos, An optimized Runge-Kutta method for the solution of orbital problems, Journal of Computational and Applied Mathematics 175(1) 1-9(2005)

82. G. Psihoyios and T.E. Simos, A fourth algebraic order trigonometrically fitted predictor-corrector scheme for IVPs with oscillating solutions, Journal of Computational and Applied Mathematics 175(1) 137-147(2005)

83. D.P. Sakas and T.E. Simos, Multiderivative methods of eighth algrebraic order with minimal phase-lag for the numerical solution of the radial Schrödinger equation, Journal of Computational and Applied Mathematics 175(1) 161$172(2005)$

84. K. Tselios and T.E. Simos, Runge-Kutta methods with minimal dispersion and dissipation for problems arising from computational acoustics, Journal of Computational and Applied Mathematics 175(1) 173-181(2005)

85. Z. Kalogiratou and T.E. Simos, Newton-Cotes formulae for long-time integration, Journal of Computational and Applied Mathematics 158(1) 75-82(2003)

86. Z. Kalogiratou, T. Monovasilis and T.E. Simos, Symplectic integrators for the numerical solution of the Schrödinger equation, Journal of Computational and Applied Mathematics 158(1) 83-92(2003)

87. A. Konguetsof and T.E. Simos, A generator of hybrid symmetric four-step methods for the numerical solution of the Schrödinger equation, Journal of Computational and Applied Mathematics 158(1) 93-106(2003)

88. G. Psihoyios and T.E. Simos, Trigonometrically fitted predictor-corrector methods for IVPs with oscillating solutions, Journal of Computational and Applied Mathematics 158(1) 135-144(2003)

89. Ch. Tsitouras and T.E. Simos, Optimized Runge-Kutta pairs for problems with oscillating solutions, Journal of Computational and Applied Mathematics 147(2) 397-409(2002)

90. T.E. Simos, An exponentially fitted eighth-order method for the numerical solution of the Schrödinger equation, Journal of Computational and Applied Mathematics 108(1-2) 177-194(1999)

91. T.E. Simos, An accurate finite difference method for the numerical solution of the Schrödinger equation, Journal of Computational and Applied Mathematics 91(1) 47-61(1998)

92. R.M. Thomas and T.E. Simos, A family of hybrid exponentially fitted predictor-corrector methods for the numerical integration of the radial 
Schrödinger equation, Journal of Computational and Applied Mathematics 87(2) 215-226(1997)

93. Z.A. Anastassi and T.E. Simos: Special Optimized Runge-Kutta methods for IVPs with Oscillating Solutions, International Journal of Modern Physics C, 15, 1-15 (2004)

94. Z.A. Anastassi and T.E. Simos: A Dispersive-Fitted and Dissipative-Fitted Explicit Runge-Kutta method for the Numerical Solution of Orbital Problems, New Astronomy, 10, 31-37 (2004)

95. Z.A. Anastassi and T.E. Simos: A Trigonometrically-Fitted Runge-Kutta Method for the Numerical Solution of Orbital Problems, New Astronomy, 10, 301-309 (2005)

96. T.V. Triantafyllidis, Z.A. Anastassi and T.E. Simos: Two Optimized RungeKutta Methods for the Solution of the Schr?dinger Equation, MATCH Commun. Math. Comput. Chem., 60, 3 (2008)

97. Z.A. Anastassi and T.E. Simos, Trigonometrically Fitted Fifth Order RungeKutta Methods for the Numerical Solution of the Schrödinger Equation, Mathematical and Computer Modelling, 42 (7-8), 877-886 (2005)

98. Z.A. Anastassi and T.E. Simos: New Trigonometrically Fitted Six-Step Symmetric Methods for the Efficient Solution of the Schrödinger Equation, MATCH Commun. Math. Comput. Chem., 60, 3 (2008)

99. G.A. Panopoulos, Z.A. Anastassi and T.E. Simos: Two New Optimized Eight-Step Symmetric Methods for the Efficient Solution of the Schrödinger Equation and Related Problems, MATCH Commun. Math. Comput. Chem., 60, 3 (2008)

100. Z.A. Anastassi and T.E. Simos: A Six-Step P-stable Trigonometrically-Fitted Method for the Numerical Integration of the Radial Schrödinger Equation, MATCH Commun. Math. Comput. Chem., 60, 3 (2008)

101. Z.A. Anastassi and T.E. Simos, A family of two-stage two-step methods for the numerical integration of the Schrödinger equation and related IVPs with oscillating solution, Journal of Mathematical Chemistry, Article in Press, Corrected Proof

102. T.E. Simos and P.S. Williams, A finite-difference method for the numerical solution of the Schrödinger equation, Journal of Computational and Applied Mathematics 79(2) 189-205(1997)

103. G. Avdelas and T.E. Simos, A generator of high-order embedded P-stable methods for the numerical solution of the Schrödinger equation, Journal of Computational and Applied Mathematics 72(2) 345-358(1996)

104. R.M. Thomas, T.E. Simos and G.V. Mitsou, A family of Numerov-type exponentially fitted predictor-corrector methods for the numerical integration of the radial Schrödinger equation, Journal of Computational and Applied Mathematics 67(2) 255-270(1996)

105. T.E. Simos, A Family of 4-Step Exponentially Fitted Predictor-Corrector Methods for the Numerical-Integration of The Schrödinger-Equation, Journal of Computational and Applied Mathematics 58(3) 337-344(1995)

106. T.E. Simos, An Explicit 4-Step Phase-Fitted Method for the NumericalIntegration of 2nd-order Initial-Value Problems, Journal of Computational and Applied Mathematics 55(2) 125-133(1994)

107. T.E. Simos, E. Dimas and A.B. Sideridis, A Runge-Kutta-Nyström Method for the Numerical-Integration of Special 2nd-order Periodic Initial-Value Problems, Journal of Computational and Applied Mathematics 51(3) 317326(1994) 
108. A.B. Sideridis and T.E. Simos, A Low-Order Embedded Runge-Kutta Method for Periodic Initial-Value Problems, Journal of Computational and Applied Mathematics 44(2) 235-244(1992)

109. T.E. Simos amd A.D. Raptis, A 4th-order Bessel Fitting Method for the Numerical-Solution of the SchrÖdinger-Equation, Journal of Computational and Applied Mathematics 43(3) 313-322(1992)

110. T.E. Simos, Explicit 2-Step Methods with Minimal Phase-Lag for the Numerical-Integration of Special 2nd-order Initial-Value Problems and their Application to the One-Dimensional Schrödinger-Equation, Journal of Computational and Applied Mathematics 39(1) 89-94(1992)

111. T.E. Simos, A 4-Step Method for the Numerical-Solution of the SchrödingerEquation, Journal of Computational and Applied Mathematics 30(3) 251255(1990)

112. C.D. Papageorgiou, A.D. Raptis and T.E. Simos, A Method for Computing Phase-Shifts for Scattering, Journal of Computational and Applied Mathematics 29(1) 61-67(1990)

113. A.D. Raptis, Two-Step Methods for the Numerical Solution of the Schrödinger Equation, Computing 28 373-378(1982).

114. T.E. Simos. A new Numerov-type method for computing eigenvalues and resonances of the radial Schrödinger equation, International Journal of Modern Physics C-Physics and Computers, 7(1) 33-41(1996)

115. T.E. Simos, Predictor Corrector Phase-Fitted Methods for $Y "=F(X, Y)$ and an Application to the Schrödinger-Equation, International Journal of Quantum Chemistry, 53(5) 473-483(1995)

116. T.E. Simos, Two-step almost P-stable complete in phase methods for the numerical integration of second order periodic initial-value problems, Inter. J. Comput. Math. 46 77-85(1992).

117. R. M. Corless, A. Shakoori, D.A. Aruliah, L. Gonzalez-Vega, Barycentric Hermite Interpolants for Event Location in Initial-Value Problems, JNAIAM J. Numer. Anal. Indust. Appl. Math, 3, 1-16 (2008)

118. M. Dewar, Embedding a General-Purpose Numerical Library in an Interactive Environment, JNAIAM J. Numer. Anal. Indust. Appl. Math, 3, 17-26 (2008)

119. J. Kierzenka and L.F. Shampine, A BVP Solver that Controls Residual and Error, JNAIAM J. Numer. Anal. Indust. Appl. Math, 3, 27-41 (2008)

120. R. Knapp, A Method of Lines Framework in Mathematica, JNAIAM J. Numer. Anal. Indust. Appl. Math, 3, 43-59 (2008)

121. N. S. Nedialkov and J. D. Pryce, Solving Differential Algebraic Equations by Taylor Series (III): the DAETS Code, JNAIAM J. Numer. Anal. Indust. Appl. Math, 3, 61-80 (2008)

122. R. L. Lipsman, J. E. Osborn, and J. M. Rosenberg, The SCHOL Project at the University of Maryland: Using Mathematical Software in the Teaching of Sophomore Differential Equations, JNAIAM J. Numer. Anal. Indust. Appl. Math, 3, 81-103 (2008)

123. M. Sofroniou and G. Spaletta, Extrapolation Methods in Mathematica, JNAIAM J. Numer. Anal. Indust. Appl. Math, 3, 105-121 (2008)

124. R. J. Spiteri and Thian-Peng Ter, pythNon: A PSE for the Numerical Solution of Nonlinear Algebraic Equations, JNAIAM J. Numer. Anal. Indust. Appl. Math, 3, 123-137 (2008)

125. S.P. Corwin, S. Thompson and S.M. White, Solving ODEs and DDEs with Impulses, JNAIAM J. Numer. Anal. Indust. Appl. Math, 3, 139-149 (2008) 
126. W. Weckesser, VFGEN: A Code Generation Tool, JNAIAM J. Numer. Anal. Indust. Appl. Math, 3, 151-165 (2008)

127. A. Wittkopf, Automatic Code Generation and Optimization in Maple, JNAIAM J. Numer. Anal. Indust. Appl. Math, 3, 167-180 (2008) 
Paper_1_SAV.tex; 30/10/2018; 15:54; p.40 


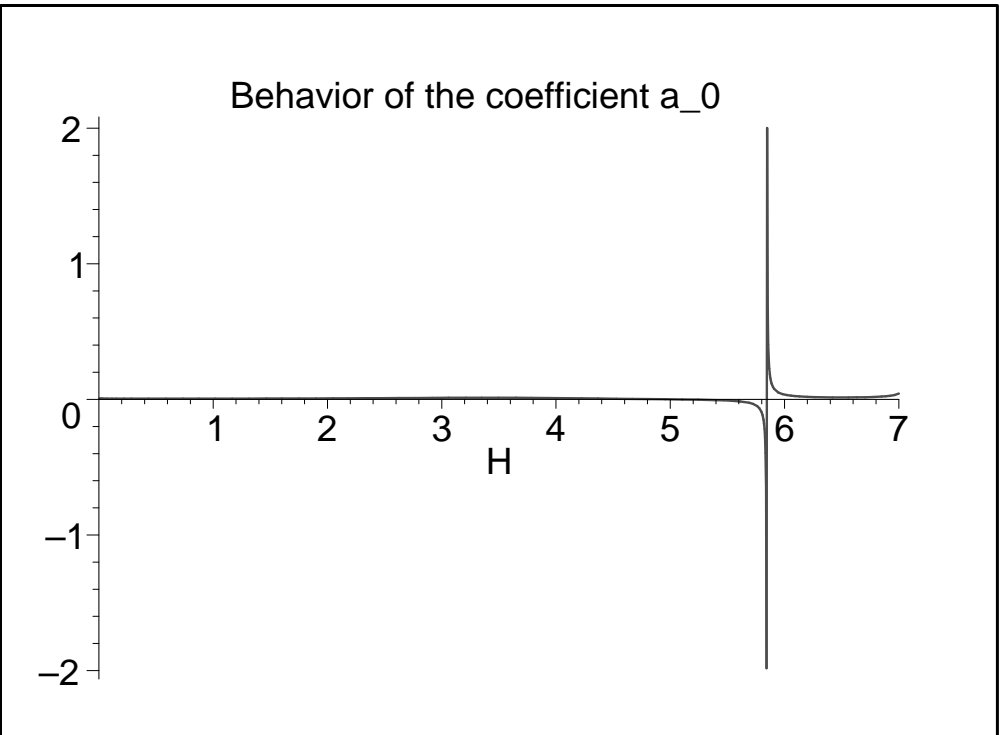




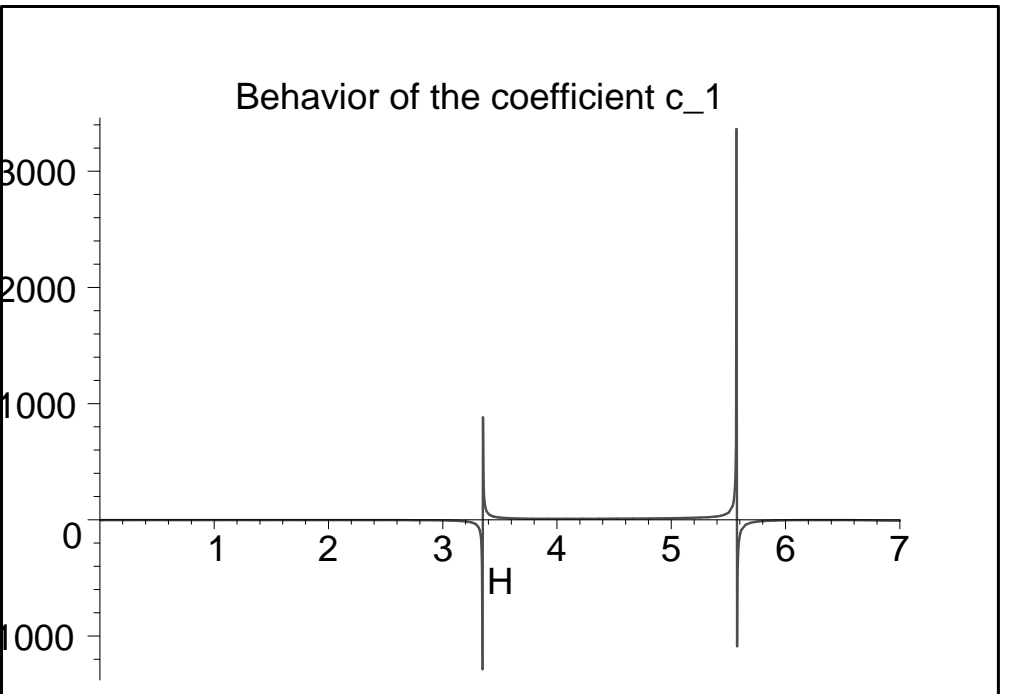




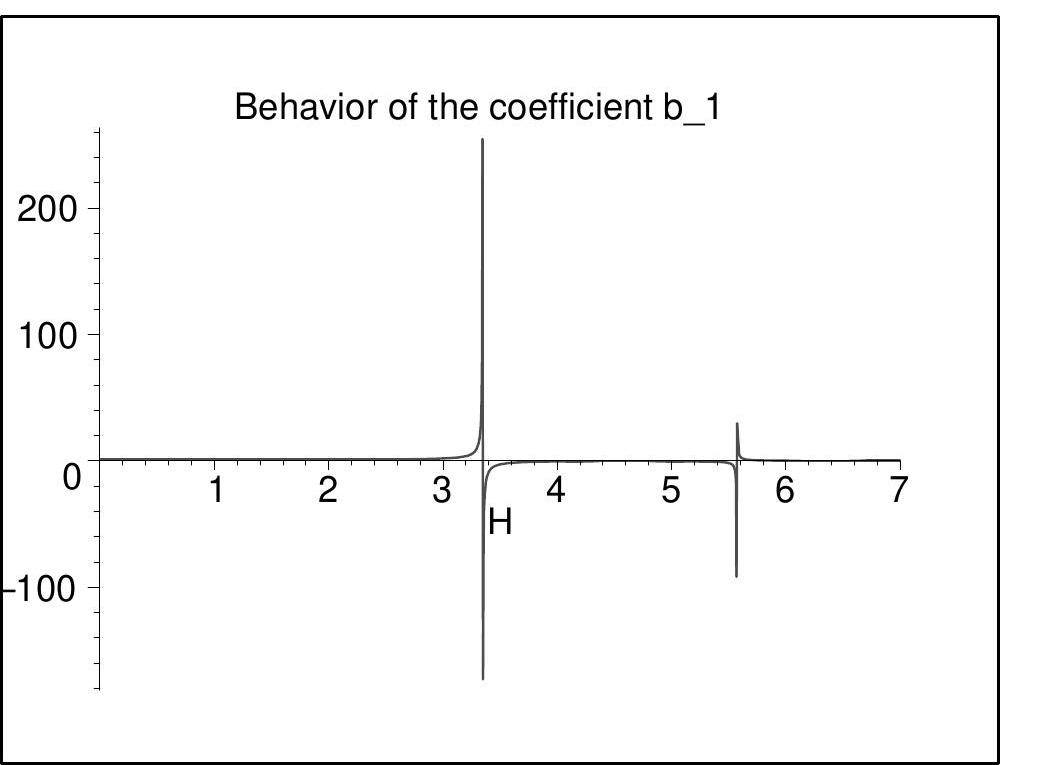




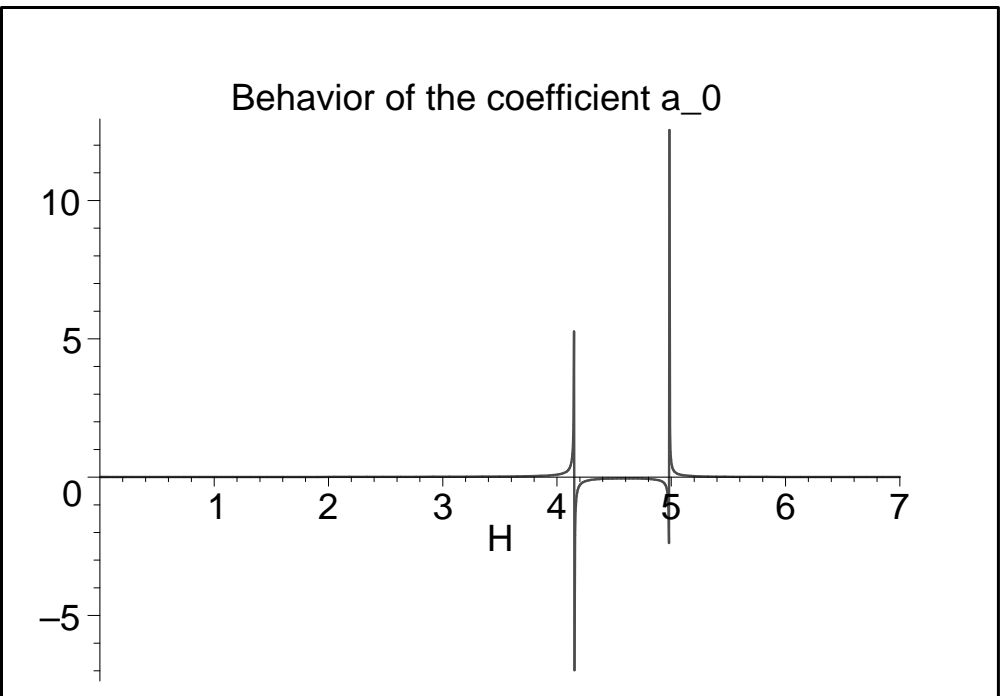




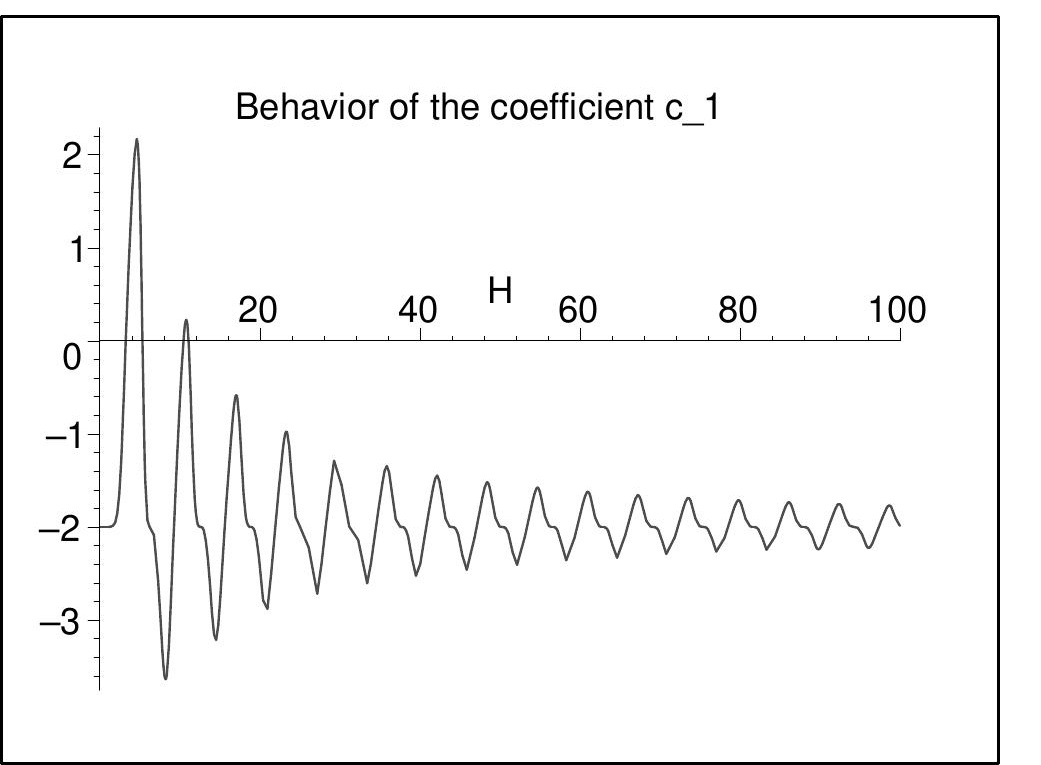




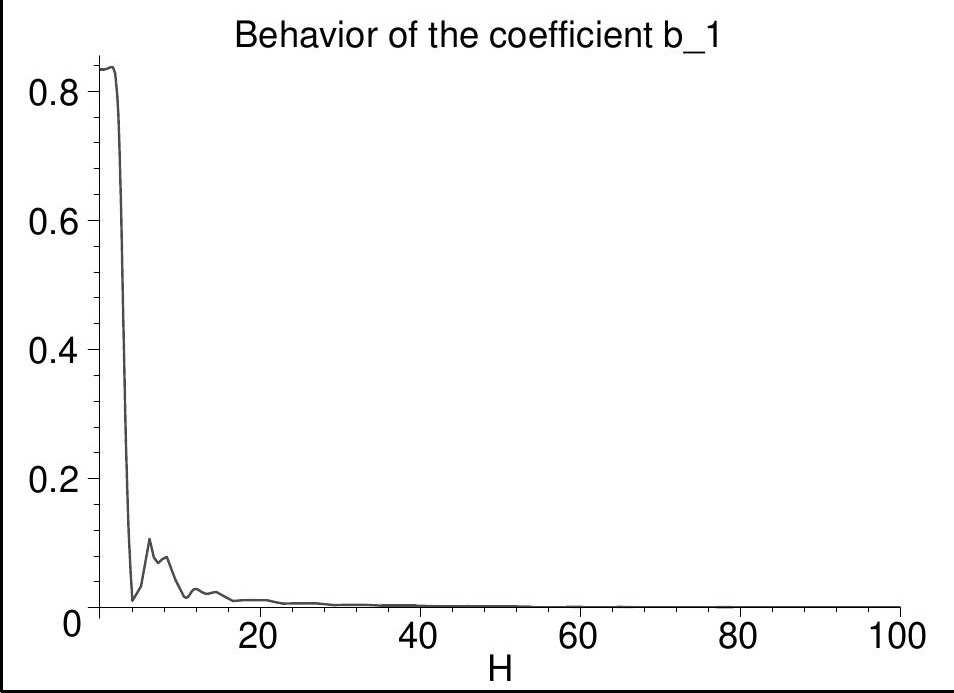




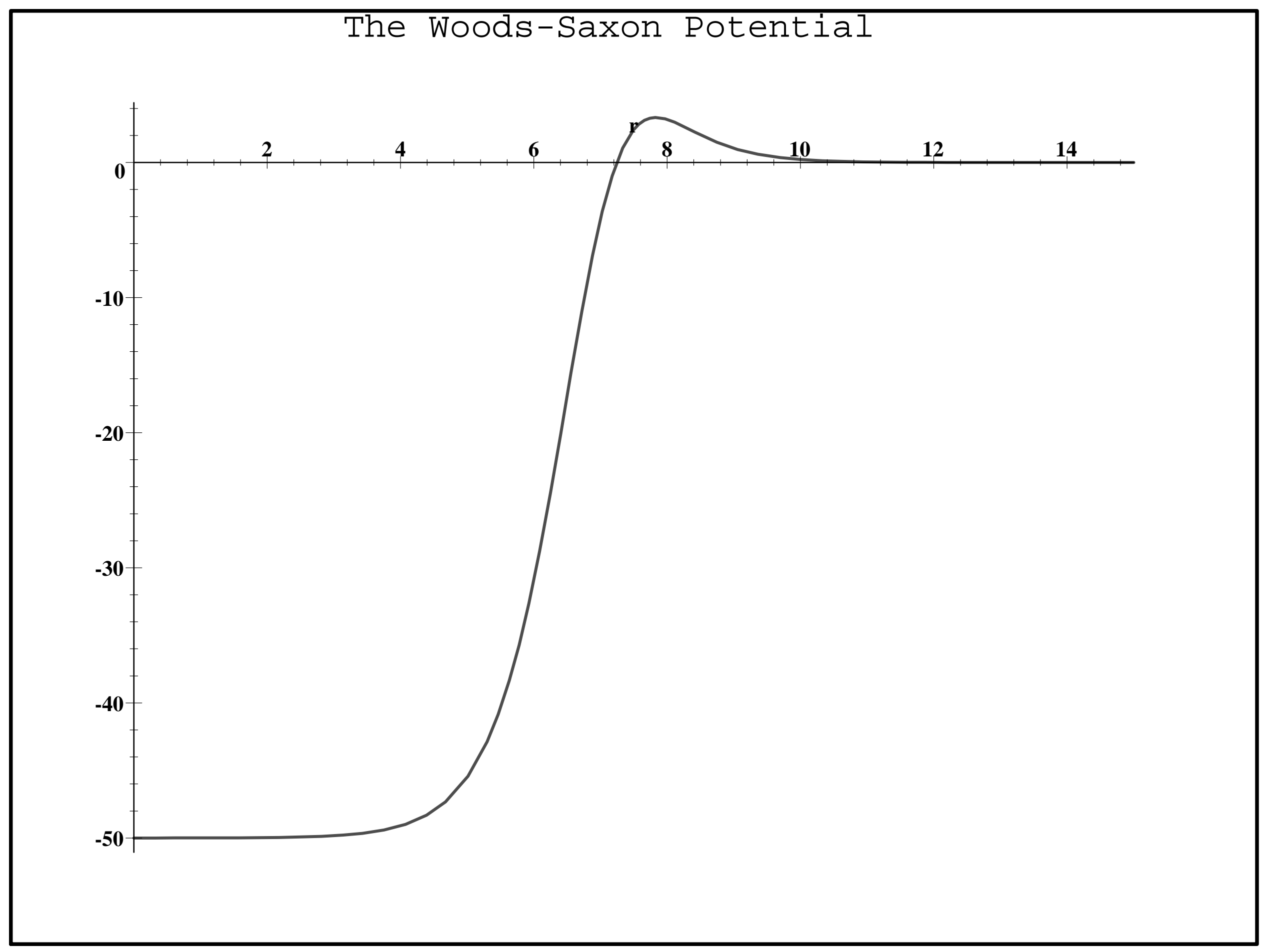

